\title{
Strong first order electroweak phase transition in 2HDM confronting future $Z$ \& Higgs factories
}

\author{
Wei Su, ${ }^{b}$ Anthony G. Williams ${ }^{b}$ and Mengchao Zhang $^{a, 1}$ \\ ${ }^{a}$ Department of Physics and Siyuan Laboratory, Jinan University, \\ Guangzhou 510632, P.R. China \\ ${ }^{b}$ ARC Centre of Excellence for Dark Matter Particle Physics, \\ Department of Physics, University of Adelaide, \\ South Australia 5005, Australia \\ E-mail: wei.su@adelaide.edu.au, anthony.williams@adelaide.edu.au, \\ mczhang@jnu.edu.cn
}

ABSTRACT: The electroweak phase transition can be made first order by extending the Standard Model (SM) Higgs sector with extra scalars. The same new physics can explain the matter-antimatter asymmetry of the universe by supplying an extra source of CP violation and sphaleron processes. In this paper we study the existence of strong first order electroweak phase transition (SFOEWPT) in the type-I and type-II two Higgs doublet models $(2 \mathrm{HDM})$. We focus on how the SFOEWPT requirements constraint the spectrum of non-SM Higgs. Through the parameter space scan, we find that SFOEWPT suggests an upper limit on the masses of heavy Higgs $m_{A / H / H^{ \pm}}$, which is around $1 \mathrm{TeV}$. High temperature expansion and Higgs vacuum uplifting is used for an analytical understanding of our results. After taking into account the probe ability on SFOEWPT from theoretical constraints, Higgs and $Z$-pole precision measurements up to the one-loop level at future Higgs \& $Z$ factories, sizeable loop corrections require $m_{A / H^{ \pm}}-m_{H} \in(100,250) \mathrm{GeV}$ to meet SFOEWPT condition for Type-II $2 \mathrm{HDM}$, and $\left|m_{A / H^{ \pm}}-m_{H}\right| \in(100,350) \mathrm{GeV}$ or $\left|m_{A}-m_{H / H^{ \pm}}\right| \in(100,350) \mathrm{GeV}$ for Type-I 2HDM.

KEYWORDS: Supersymmetry Phenomenology

ARXIV EPRINT: 2011.04540

\footnotetext{
${ }^{1}$ Corresponding author
} 


\section{Contents}

1 Introduction 1

2 The electroweak phase transition in 2HDMs 3

2.1 Two Higgs doublet models 3

2.2 Thermal effective potential 4

2.3 Numerical analysis method 6

3 Current and expected bounds $\quad 7$

3.1 Theoretical constraints 8

3.2 Direct searches at LHC Run-II 9

$\begin{array}{lll}3.3 & \text { Higgs and } Z \text { pole precision measurements } & 10\end{array}$

$\begin{array}{lll}3.4 & \text { Flavour constraints } & 11\end{array}$

4 Study results $\quad 11$

4.1 The phase transition of 2HDM 11

4.1.1 High temperature expansion 11

$\begin{array}{lll}\text { 4.1.2 Higgs vacuum uplifting } & 13\end{array}$

4.2 Case1: alignment limit with $m_{A / H^{ \pm}}-m_{H}=200 \mathrm{GeV} \quad 17$

$\begin{array}{ll}4.3 \text { Case2: alignment limit with } m_{A}=m_{H^{ \pm}} & 18\end{array}$

$\begin{array}{ll}\text { 4.4 Case3: alignment limit with } m_{H}=700 \mathrm{GeV} & 19\end{array}$

4.5 General results 20

5 Conclusion $\quad 24$

\section{Introduction}

The discovery of the Higgs boson in 2012 completes the Standard Model (SM) [1, 2], yet there remain observations that cannot be explained by it. One of the most famous puzzles is the baryon asymmetry of the universe (BAU), which sees the visible matter in our universe being dominated by baryons whilst the amount of anti-baryons is negligible. Particle physics models that can successfully explain the BAU need to satisfy the three Sakharov conditions [3]. The SM was once considered as a candidate model [4-6], since baryon number conservation can be broken by an electroweak sphaleron process $[4,7,8]$, the CKM matrix provides CP violation, and the electroweak phase transition can induce a departure from equilibrium if the Higgs boson is light enough. However, such an electroweak baryogenesis (EWBG) mechanism in the SM framework turns out to fail, since the CP violation present in the CKM matrix is too small [9] and the measured Higgs mass is too heavy to trigger a strong first order electroweak phase transition (SFOEWPT) [10, 11]. 
Thus for a successful baryogenesis, new physics beyond the SM (BSM) is required to supply a new source of $\mathrm{CP}$ violation and a strong out-of-equilibrium process $[12,13]$. In this work we focus on the latter issue.

In order to obtain a SFOEWPT, generally we need to extend the scalar sector of the SM. Additional parameters in the scalar sector help to change the shape of Higgs potential whilst leaving the Higgs vacuum expectation value (VEV) and the mass of Higgs same. Simple SM extensions include the addition of a $\mathrm{SU}(2)$ singlet [14-31], an extra doublet [32-69], an extra triplet [70-73], or extra higher dimensional operators [74-79]. Consideration of the hierarchy problem leads to further solutions such as embedding the Higgs boson in a Composite Higgs model [80-82] or a supersymmetric model [83-98] to obtain a SFOEWPT. For a SFOEWPT in other models see [99-102]. In this work we study the existence of a SFOEWPT in the type-I and type-II 2HDMs [103, 104]. These models are attractive to study because the number of new parameters is relatively small. In addition, both Higgs doublets in $2 \mathrm{HDM}$ models are charged under $\mathrm{SU}(2) \times \mathrm{U}(1)$ and couple to SM fermions. This gives a greater range of observations that can probe the models relative to models that are extended by SM singlet scalars.

It is well known that, compared with other baryogenesis mechanisms, e.g. leptogenesis [105] or the Affleck-Dine mechanism [106], EWBG can be detected at the electroweak scale and part of the parameter space can be covered by current or expected collider experiments. In 2HDMs, in addition to the SM-like Higgs boson $h$, there are three non-SM Higgs bosons, $H / A / H^{ \pm} . H / A / H^{ \pm}$couple to $h$ and help to build an energy barrier between the symmetric phase and the $\mathrm{SU}(2) \times \mathrm{U}(1)$ broken phase when the temperature of the universe is around the electroweak scale. Then the phase transition, which is tunneling through the energy barrier, can be first order and strong enough. In our study, we will show that, in order for this strong first order phase transition to occur, the masses of $H, A$ and $H^{ \pm}$ bosons should all be smaller than about $1 \mathrm{TeV}$, and generally there needs to be a relatively large mass splitting between the heavy Higgs bosons $H, A$ and $H^{ \pm}$.

$H, A$, and $H^{ \pm}$bosons with a mass lighter than $1 \mathrm{TeV}$ can be directly produced at the current LHC or future hadron colliders like the HE-LHC [107] or the SPPC [108, 109]. Channels like $A / H \rightarrow t \bar{t} / b \bar{b} / \tau \bar{\tau}, H^{ \pm} \rightarrow t \bar{b}$, or $A \rightarrow H Z$ [110-113] can be used for detection or exclusion. Besides, through mixing and loop effects, the non-SM Higgs bosons also change the predicted value of the oblique parameters $S, T$ and $U$, and reduce the Higgs couplings $\kappa_{i}=g_{h} i i^{2 \mathrm{HDM}} / g_{h} i i^{\mathrm{SM}}$ relative to the SM expectation. Future $e^{+} e^{-}$colliders like the ILC [114], FCC-ee [115, 116] and CEPC [108, 109] will copiously produce $Z$ and Higgs bosons, and thus those observables (especially the $h Z Z$ coupling) can be measured with unprecedented precision. In this work, we perform a global fit to obtain the parameter space of $2 \mathrm{HDM}$ s that simultaneously satisfies a SFOEWPT and the expected measurement precision at future $Z$ and Higgs factories.

The structure of this paper is as follows. In section 2 we briefly introduce our 2HDM models and calculation methods. In section 3 we list all relevant measurements that can be used to constrain the parameter space of the type-I and type-II 2HDMs. Section 4 starts with an analytic analysis which helps readers to understand the features of the electroweak phase transition in $2 \mathrm{HDMs}$. Then we study three simplified typical cases, and present the most general scan result. We conclude this work in section 5 . 


\section{The electroweak phase transition in $2 \mathrm{HDMs}$}

\subsection{Two Higgs doublet models}

2 HDMs without a $\mathbb{Z}_{2}$ symmetry generally induce dangerous flavour-violating couplings at tree level. In this work we therefore consider $2 \mathrm{HDMs}$ with a soft $\mathbb{Z}_{2}$ symmetry breaking. The tree-level scalar potential for a $2 \mathrm{HDM}$ can be written as:

$$
\begin{aligned}
V^{0}\left(\Phi_{1}, \Phi_{2}\right)= & m_{11}^{2} \Phi_{1}^{\dagger} \Phi_{1}+m_{22}^{2} \Phi_{2}^{\dagger} \Phi_{2}-m_{12}^{2}\left(\Phi_{1}^{\dagger} \Phi_{2}+\text { h.c. }\right)+\frac{\lambda_{1}}{2}\left(\Phi_{1}^{\dagger} \Phi_{1}\right)^{2}+\frac{\lambda_{2}}{2}\left(\Phi_{2}^{\dagger} \Phi_{2}\right)^{2} \\
& +\lambda_{3}\left(\Phi_{1}^{\dagger} \Phi_{1}\right)\left(\Phi_{2}^{\dagger} \Phi_{2}\right)+\lambda_{4}\left(\Phi_{1}^{\dagger} \Phi_{2}\right)\left(\Phi_{2}^{\dagger} \Phi_{1}\right)+\frac{\lambda_{5}}{2}\left[\left(\Phi_{1}^{\dagger} \Phi_{2}\right)^{2}+\text { h.c. }\right] .
\end{aligned}
$$

We consider a CP-conserving case, in which all mass parameters $m_{i j}^{2}$ and quartic couplings $\lambda_{i}$ are real. After electroweak symmetry breaking (EWSB), the two $\mathrm{SU}(2)_{L}$ Higgs doublets $\Phi_{i}$ obtain VEVs $v_{i}$, and they can be expanded in the component real scalar fields:

$$
\Phi_{1}=\left(\begin{array}{c}
\phi_{1}^{+} \\
\frac{1}{\sqrt{2}}\left(v_{1}+h_{1}+i a_{1}\right)
\end{array}\right) \quad, \quad \Phi_{2}=\left(\begin{array}{c}
\phi_{2}^{+} \\
\frac{1}{\sqrt{2}}\left(v_{2}+h_{2}+i a_{2}\right)
\end{array}\right) .
$$

with $v_{1}^{2}+v_{2}^{2} \equiv v^{2} \approx(246 \mathrm{GeV})^{2}$. We further define the ratio of VEVs as $\tan \beta \equiv v_{2} / v_{1}$.

Two of the three $m_{i j}^{2}$ can be replaced by other parameters by imposing conditions that result from minimising the Higgs potential

$$
\begin{aligned}
& m_{11}^{2}=m_{12}^{2} \frac{v_{2}}{v_{1}}-\frac{v_{1}^{2}}{2} \lambda_{1}-\frac{v_{2}^{2}}{2}\left(\lambda_{3}+\lambda_{4}+\lambda_{5}\right) \\
& m_{22}^{2}=m_{12}^{2} \frac{v_{1}}{v_{2}}-\frac{v_{2}^{2}}{2} \lambda_{2}-\frac{v_{1}^{2}}{2}\left(\lambda_{3}+\lambda_{4}+\lambda_{5}\right) .
\end{aligned}
$$

Thus the squared mass matrices of the CP-even, CP-odd, and charged Higgs are:

$$
\begin{aligned}
\mathcal{M}_{\text {even }}^{2} & =\left(\begin{array}{ll}
m_{12}^{2} \tan \beta+\lambda_{1} v_{1}^{2} & -m_{12}^{2}+v_{1} v_{2} \lambda_{345} \\
-m_{12}^{2}+v_{1} v_{2} \lambda_{345} & m_{12}^{2} / \tan \beta+\lambda_{2} v_{2}^{2}
\end{array}\right), \\
\mathcal{M}_{\text {odd }}^{2} & =\left(m_{12}^{2}-v_{1} v_{2} \lambda_{5}\right)\left(\begin{array}{cc}
\tan \beta & -1 \\
-1 & 1 / \tan \beta
\end{array}\right), \\
\mathcal{M}_{\text {charged }}^{2} & =\left(m_{12}^{2}-\frac{1}{2} v_{1} v_{2}\left(\lambda_{4}+\lambda_{5}\right)\right)\left(\begin{array}{cc}
\tan \beta & -1 \\
-1 & 1 / \tan \beta
\end{array}\right) .
\end{aligned}
$$

Here $\lambda_{345} \equiv \lambda_{3}+\lambda_{4}+\lambda_{5}$. After diagonalization, the mass eigenstates are related to the original fields by the rotation matrices:

$$
\begin{aligned}
\left(\begin{array}{c}
H \\
h
\end{array}\right) & =\left(\begin{array}{cc}
\cos \alpha & \sin \alpha \\
-\sin \alpha & \cos \alpha
\end{array}\right)\left(\begin{array}{l}
h_{1} \\
h_{2}
\end{array}\right), \\
\left(\begin{array}{c}
G^{0} \\
A
\end{array}\right) & =\left(\begin{array}{cc}
\cos \beta & \sin \beta \\
-\sin \beta & \cos \beta
\end{array}\right)\left(\begin{array}{l}
a_{1} \\
a_{2}
\end{array}\right), \\
\left(\begin{array}{c}
G^{ \pm} \\
H^{ \pm}
\end{array}\right) & =\left(\begin{array}{cc}
\cos \beta & \sin \beta \\
-\sin \beta & \cos \beta
\end{array}\right)\left(\begin{array}{l}
\phi_{1}^{ \pm} \\
\phi_{2}^{ \pm}
\end{array}\right)
\end{aligned}
$$


We choose our input parameters to be:

$$
\cos (\beta-\alpha), \tan \beta, m_{12}^{2}, m_{H}, m_{A}, m_{H^{ \pm}} .
$$

The mass of the SM-like Higgs boson $m_{h}$ is fixed to the current central measured value $125.09 \mathrm{GeV}$ [117]. Then the $\lambda_{i}$ can be re-expressed in terms of these input parameters. Considering the theoretical constraints, including vacuum stability, perturbativity, and unitarity, we introduce

$$
\lambda v^{2} \equiv m_{H}^{2}-\frac{m_{12}^{2}}{\sin \beta \cos \beta},
$$

following the notation in [118]. Under the assumption of degenerate heavy Higgs masses $m_{H}=m_{A}=m_{H^{ \pm}}$, there is no theoretical restriction on the $\tan \beta$ range when $\sqrt{\lambda v^{2}}=0$.

Type-I and Type-II 2 HDMs have different $\mathbb{Z}_{2}$ parity assignments, and thus the couplings between scalar and other particles have a different dependence on $\tan \beta$ and the mixing angle $\alpha$. The main difference between the Type I and Type II models is the dependence of the couplings $A f \bar{f}$ and $H f \bar{f}$ on the value of $\tan \beta$. Couplings between $A / H$ and down-type fermions are suppressed by $\frac{1}{\tan \beta}$ in the Type I model, but are enhanced by $\tan \beta$ in the Type II model. Thus the Type II model is generally more constrained by experiments than the Type I model when $\tan \beta$ is large.

Here we need to emphasize that in the $2 \mathrm{HDM}$ we can set the mass of the non-SM Higgs bosons $A / H / H^{ \pm}$to an arbitrarily high scale. This is because of the presence of $m_{11,12,22}^{2}$, with $m_{12}^{2}$ breaking $\mathbb{Z}_{2}$ symmetry in eq. (2.1). As can be seen from eq. (2.5) to eq. (2.7), the squared masses of $A / H / H^{ \pm}$arise from two types of contribution. One of them involves terms of the form $\lambda_{i} v_{j} v_{k}$, which are bounded by perturbative unitarity and thus cannot be too large. Upper-limits on these terms are roughly given by $4 \pi v^{2} \approx(870 \mathrm{GeV})^{2}$. Another part of Higgs mass squares come from $m_{12}^{2}\left(m_{11 / 22}^{2}\right.$ are transformed through eq. (2.3) and eq. (2.4)), and these terms can in principle be set to any value without violating theoretical requirements. This makes the search for evidence of $2 \mathrm{HDMs}$ an endless game: you can never completely falsify a New Physics model containing hypothetical particles which have no upper limits on their mass.

However, in the following part of this work we will show that the requirement of a SFOEWPT imposes upper limits on the masses of the $A / H / H^{ \pm}$bosons, making it possible to fully verify or falsify the idea of EWBG in $2 \mathrm{HDMs}$ in the near future.

\subsection{Thermal effective potential}

To study the phase transition in the early universe, we need to study the dependence of the free energy density on the order parameter. In our case, the free energy density is the thermal effective potential, and the order parameter is the homogeneous scalar VEV [119]. The thermal effective potential $V\left(\phi_{1}, \phi_{2}, T\right)$ at temperature $T$ is composed of four parts:

$$
V\left(\phi_{1}, \phi_{2}, T\right)=V^{0}\left(\phi_{1}, \phi_{2}\right)+V^{\mathrm{CW}}\left(\phi_{1}, \phi_{2}\right)+V^{\mathrm{CT}}\left(\phi_{1}, \phi_{2}\right)+V^{\mathrm{T}}\left(\phi_{1}, \phi_{2}, T\right) .
$$

Here $V^{0}$ is the tree-level potential of our model, $V^{\mathrm{CW}}$ is one-loop Coleman-Weinberg potential, $V^{\mathrm{CT}}$ is the counter term, and $V^{\mathrm{T}}$ is the thermal correction. 
The tree-level potential $V^{0}\left(\phi_{1}, \phi_{2}\right)$ is obtained by replacing the field operators $\Phi_{1}(x)$ and $\Phi_{2}(x)$ in $V^{0}\left(\Phi_{1}, \Phi_{2}\right)$ with the homogeneous field values $\frac{1}{\sqrt{2}}\left(0, \phi_{1}\right)^{T}$ and $\frac{1}{\sqrt{2}}\left(0, \phi_{2}\right)^{T}$ :

$$
V^{0}\left(\phi_{1}, \phi_{2}\right)=\frac{1}{2} m_{11}^{2} \phi_{1}^{2}+\frac{1}{2} m_{22}^{2} \phi_{2}^{2}-m_{12}^{2} \phi_{1} \phi_{2}+\frac{1}{8} \lambda_{1} \phi_{1}^{4}+\frac{1}{8} \lambda_{2} \phi_{2}^{4}+\frac{1}{4} \lambda_{345} \phi_{1}^{2} \phi_{2}^{2} .
$$

The one-loop Coleman-Weinberg potential $V^{\mathrm{CW}}\left(\phi_{1}, \phi_{2}\right)$ is given in the $\overline{\mathrm{MS}}$ renormalization scheme by [120]:

$$
V^{\mathrm{CW}}\left(\phi_{1}, \phi_{2}\right)=\frac{1}{64 \pi^{2}} \sum_{i} n_{i} m_{i}^{4}\left(\phi_{1}, \phi_{2}\right)\left[\ln \frac{m_{i}^{2}\left(\phi_{1}, \phi_{2}\right)}{\mu^{2}}-c_{i}\right],
$$

with the index $i$ running over all massive particles. $n_{i}$ is the degrees of freedom of particle $i$ multiplied by $(-1)^{2 s}$ ( $s$ is the spin of particle $i$ ), which is $-12,-4,6,3,2,1,2$ and 1 for quarks, leptons, $W^{ \pm}, Z, H^{ \pm}, G^{0}, G^{ \pm}$, and neutral scalars, respectively. $c_{i}$ is $\frac{5}{6}$ for gauge bosons, and $\frac{3}{2}$ for other particles. $m_{i}^{2}\left(\phi_{1}, \phi_{2}\right)$ is the mass square of particle $i$ with $v_{1}$ and $v_{2}$ in its expression being replaced by scalar field value $\phi_{1}$ and $\phi_{2}$. The renormalization scale $\mu$ is set to the zero temperature VEV $v$.

In eq. (2.11) we choose the scalar masses, mixing angle, and VEV ratio as our input parameters. These parameters are considered as physical parameters. It means that the VEVs are determined by the position of the minimum of the scalar potential, and squared masses are given by the second order partial derivatives of the scalar potential with respect to the scalar fields at the position of the minimum. Adding the Coleman-Weinberg correction will shift both the position of the minimum and the second order partial derivatives of the tree-level potential.

Thus, in order to offset the modification, counter terms $V^{\mathrm{CT}}\left(\Phi_{1}, \Phi_{2}\right)$ need to be added to the Lagrangian. For a CP-conserving $2 \mathrm{HDM}, V^{\mathrm{CT}}\left(\Phi_{1}, \Phi_{2}\right)$ can be expressed as [52]:

$$
\begin{aligned}
V^{\mathrm{CT}}\left(\Phi_{1}, \Phi_{2}\right)= & \delta m_{11}^{2} \Phi_{1}^{\dagger} \Phi_{1}+\delta m_{22}^{2} \Phi_{2}^{\dagger} \Phi_{2}-\delta m_{12}^{2}\left(\Phi_{1}^{\dagger} \Phi_{2}+\text { h.c. }\right)+\frac{\delta \lambda_{1}}{2}\left(\Phi_{1}^{\dagger} \Phi_{1}\right)^{2}+\frac{\delta \lambda_{2}}{2}\left(\Phi_{2}^{\dagger} \Phi_{2}\right)^{2} \\
& +\delta \lambda_{3}\left(\Phi_{1}^{\dagger} \Phi_{1}\right)\left(\Phi_{2}^{\dagger} \Phi_{2}\right)+\delta \lambda_{4}\left(\Phi_{1}^{\dagger} \Phi_{2}\right)\left(\Phi_{2}^{\dagger} \Phi_{1}\right)+\frac{\delta \lambda_{5}}{2}\left[\left(\Phi_{1}^{\dagger} \Phi_{2}\right)^{2}+\text { h.c. }\right] \\
& +\delta t_{1} \phi_{1}+\delta t_{2} \phi_{2} .
\end{aligned}
$$

Coefficients of counter terms, those $\delta$ s, need to be fixed by "on-shell" conditions:

$$
\begin{array}{r}
\partial_{\psi_{i}}\left(V^{\mathrm{CT}}\left(\Phi_{1}, \Phi_{2}\right)+V^{\mathrm{CW}}\left(\Phi_{1}, \Phi_{2}\right)\right)=0 \\
\partial_{\psi_{i}} \partial_{\psi_{j}}\left(V^{\mathrm{CT}}\left(\Phi_{1}, \Phi_{2}\right)+V^{\mathrm{CW}}\left(\Phi_{1}, \Phi_{2}\right)\right)=0
\end{array}
$$

with $\psi_{i}$ denoting all of the component scalar fields of $\Phi_{1}$ and $\Phi_{2}$. These conditions are evaluated at the minimum of the scalar potential at zero temperature, where $\Phi_{1}=\frac{1}{\sqrt{2}}\left(0, v_{1}\right)^{T}$ and $\Phi_{2}=\frac{1}{\sqrt{2}}\left(0, v_{2}\right)^{T}{ }^{1}$ After adding these counter terms, our input parameters can be treated as physical parameters which are directly connected to observables.

\footnotetext{
${ }^{1}$ Second order derivatives of $V^{\mathrm{CW}}$ suffer from an infrared divergence originating from the massless Goldstone boson when $T=0$. This problem can be solved by introducing an IR cut-off mass [50].
} 
The thermal correction with ring resummation included is [121, 122]:

$$
\begin{aligned}
V^{T}\left(\phi_{1}, \phi_{2}, T\right)= & \frac{T^{4}}{2 \pi^{2}} \sum_{i} n_{i} J_{B}\left(\frac{m_{i}^{2}\left(\phi_{1}, \phi_{2}\right)}{T^{2}}\right)+\frac{T^{4}}{2 \pi^{2}} \sum_{j} n_{j} J_{F}\left(\frac{m_{j}^{2}\left(\phi_{1}, \phi_{2}\right)}{T^{2}}\right) \\
& -\frac{T^{4}}{12 \pi} \sum_{k} n_{k}\left[\left(\frac{\tilde{m}_{k}^{2}\left(\phi_{1}, \phi_{2}, T\right)}{T^{2}}\right)^{3 / 2}-\left(\frac{m_{k}^{2}\left(\phi_{1}, \phi_{2}\right)}{T^{2}}\right)^{3 / 2}\right] .
\end{aligned}
$$

Here, the index $i$ denotes all gauge bosons and scalars, $j$ denotes leptons and quarks, and $k$ denotes scalars and the longitudinal component of gauge bosons. The functions $J_{B, F}$ are two integrals which come from the scalar and fermion thermal corrections respectively:

$$
\begin{aligned}
& J_{B}(x)=\int_{0}^{\infty} d k k^{2} \ln \left[1-\exp \left(-\sqrt{k^{2}+x}\right)\right] \\
& J_{F}(x)=\int_{0}^{\infty} d k k^{2} \ln \left[1+\exp \left(-\sqrt{k^{2}+x}\right)\right] .
\end{aligned}
$$

The second line in (2.19) comes from ring resummation, which is used to avoid the infrared divergence that occurs when the scalar mass is much smaller than the temperature. $\tilde{m}_{k}^{2}\left(\phi_{1}, \phi_{2}, T\right)$ is the thermal Debye mass, an expression for which can be found in the literature $[52,122]$.

\subsection{Numerical analysis method}

An electroweak phase transition is considered to be strong enough only if the net baryon number generated around the bubble wall is not significantly washed out by the sphaleron process inside the bubble. This condition can be converted to the requirement on the value of "wash out" parameter [123]:

$$
\xi_{c} \equiv \frac{v_{c}}{T_{c}}>0.9
$$

Here $T_{c}$ is the critical temperature where a second minimum of $V\left(\phi_{1}, \phi_{2}, T\right)$ that breaks $\mathrm{SU}(2) \times \mathrm{U}(1)$ appears, and $v_{c} \equiv \sqrt{v_{1}^{2}\left(T_{c}\right)+v_{2}^{2}\left(T_{c}\right)}$ reflects the scale of electroweak symmetry breaking. Here $v_{1}\left(T_{c}\right)$ and $v_{2}\left(T_{c}\right)$ are the scalar field values which minimize $V\left(\phi_{1}, \phi_{2}, T_{c}\right)$.

The calculation of $\xi_{c}$ suffers from theoretical uncertainties. The first problem is that the $\xi_{c}$ induced by $V\left(\phi_{1}, \phi_{2}, T\right)$ is not gauge independent by itself [124-126]. Missing higherorder quantum corrections also induce a theoretical uncertainty [127]. For a concrete model, one can use lattice simulations to obtain a reliable value of $\xi_{c}$ [63], but such a nonperturbative calculation is very computationally expensive. Being aware of the theoretical uncertainty in the calculation of $\xi_{c}$, in this work we relax the criterion of a SFOEWPT to $\xi_{c} \equiv \frac{v_{c}}{T_{c}}>0.9$. On the other hand, for a first order phase transition to really happen in the universe, the bubble nucleation rate should be larger than the Hubble expansion rate at the nucleation temperature [33, 128]. This requirement can be considered as a further constraint on the 2HDM parameter space. For a conservative estimate, in this work we will not consider a requirement on the bubble nucleation rate. 
Analytically, $T_{c}$ and $v_{c}$ can be obtained by solving the following equations:

$$
\begin{aligned}
V\left(0,0, T_{c}\right) & =V\left(v_{1}\left(T_{c}\right), v_{2}\left(T_{c}\right), T_{c}\right), \\
\left.\frac{\partial}{\partial \phi_{1}} V\left(\phi_{i}, \phi_{2}, T_{c}\right)\right|_{\phi_{1}=v_{1}\left(T_{c}\right), \phi_{2}=v_{2}\left(T_{c}\right)} & =0(i=1,2), \\
\left.\frac{\partial}{\partial \phi_{1}} V\left(\phi_{i}, \phi_{2}, T_{c}\right)\right|_{\phi_{1}=0, \phi_{2}=0} & =0(i=1,2) .
\end{aligned}
$$

To make $(0,0)$ and $\left(v_{1}\left(T_{c}\right), v_{2}\left(T_{c}\right)\right)$ as local minimum points of $V\left(\phi_{i}, \phi_{2}, T_{c}\right)$, Hessian matrix of $V\left(\phi_{i}, \phi_{2}, T_{c}\right)$ at $(0,0)$ and $\left(v_{1}\left(T_{c}\right), v_{2}\left(T_{c}\right)\right)$ also need to be positive definite. However, due to the complicated form of $V\left(\phi_{1}, \phi_{2}, T\right)$, solving these equations analytically is quite difficult. Instead, one can search for the critical temperature using a numerical method. There are already public packages which can be used for numerical thermal phase transition analysis, such as CosmoTransitions [129], BSMPT [61], and PhaseTracer [130]. We choose BSMPT for our numerical analysis, since the 2HDM has been implemented in BSMPT as a benchmark model, and BSMPT is written in C++ which helps to save numerical calculation time. In BSMPT, the search for $T_{c}$ is started from a high temperature (the default value is $300 \mathrm{GeV})$, where the minimum position of $V\left(\phi_{1}, \phi_{2}, T\right)$ is $(0,0)$. Then BSMPT traces the minimum position of $V\left(\phi_{1}, \phi_{2}, T\right)$ with decreasing temperature. If BSMPT detects a minimum position jumping $(0,0) \Rightarrow\left(v_{1}\left(T^{\prime}\right), v_{2}\left(T^{\prime}\right)\right)$ at a certain temperature $T^{\prime}$, the search stops and the output $T^{\prime}$ is the desired critical temperature $T_{c}$.

The full thermal phase transition history of the 2HDM could be complicated [59]. Multiple phase transition processes are possible. For baryogenesis, however, only the phase transition that transfers $(0,0) \Rightarrow\left(v_{1}(T), v_{2}(T)\right)$ is relevant. This is because a successful baryogenesis requires the sphaleron rate to be very fast outside the bubble wall, i.e. $\Gamma_{\mathrm{Sph}} \sim\left(\alpha_{W} T\right)^{4}$. While in the electroweak symmetry breaking phase, the sphaleron rate will be strongly suppressed as $\Gamma_{\mathrm{Sph}} \propto \exp \left(-E_{S p h}\left(T_{c}\right) / T_{c}\right)$. Here the sphaleron energy $E_{S p h}\left(T_{c}\right) \sim 10 \mathrm{TeV} \times \frac{v_{c}}{v}$. Thus another phase transition $\left(v_{1}\left(T^{\prime \prime}\right), v_{2}\left(T^{\prime \prime}\right)\right) \Rightarrow\left(w_{1}\left(T^{\prime \prime}\right), w_{2}\left(T^{\prime \prime}\right)\right)$ has nothing to do with baryogenesis, because the sphaleron rate outside the bubble will be too low to generate baryon number. ${ }^{2}$ We will therefore not take this kind of phase transition into account in this work.

\section{Current and expected bounds}

$2 \mathrm{HDMs}$ are constrained by various theoretical considerations and experimental measurements, such as vacuum stability, perturbativity and unitarity, as well as heavy flavor observations [131], electroweak precision measurements, and LHC Higgs measurements and non-SM Higgs searches [132]. We briefly summarize below the constraints we adopt in the following sections.

\footnotetext{
${ }^{2}$ Strictly speaking, phase transition $\left(v_{1}\left(T^{\prime \prime}\right), v_{2}\left(T^{\prime \prime}\right)\right) \Rightarrow\left(w_{1}\left(T^{\prime \prime}\right), w_{2}\left(T^{\prime \prime}\right)\right)$ may produce enough baryon number if $\sqrt{v_{1}^{2}\left(T^{\prime \prime}\right)+v_{2}^{2}\left(T^{\prime \prime}\right)}$ is very small and this phase transition is strong first order. But the possibility to obtain such a parameter point is very low in a random scan. So we ignore this case.
} 

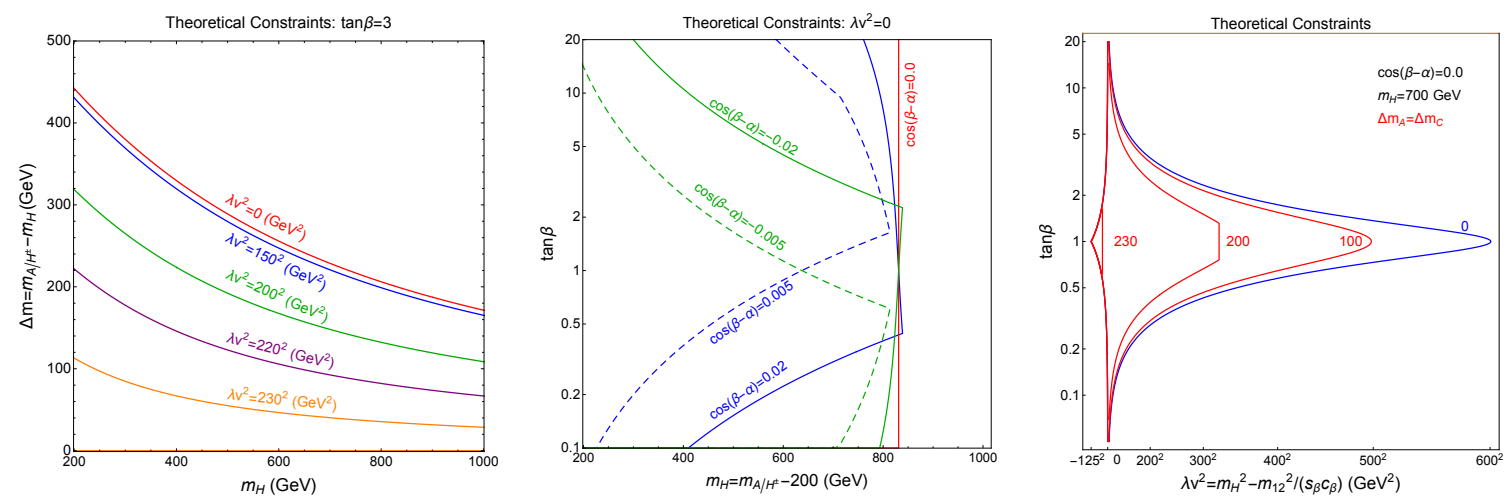

Figure 1. The impact of theoretical constraints in the $m_{H}-\Delta m$ (left), $m_{H}-\tan \beta$ (middle), $\lambda v^{2}-\tan \beta$ (right) planes. In the left panel, the allowed region is under the lines with $\tan \beta=3, \cos (\beta-\alpha)=0$. In the middle panel, $\sqrt{\lambda v^{2}}=0 \mathrm{GeV}$, and the allowed region is to the left of the corresponding lines. In the right panel, $m_{H}$ is fixed at $700 \mathrm{GeV}$, and the allowed region is inside of the boundary line. See text for full details.

\subsection{Theoretical constraints}

- Vacuum stability.

In order to make the vacuum stable, the scalar potential should be bounded from below [133-136]:

$$
\lambda_{1}>0, \lambda_{2}>0, \lambda_{3}>-\sqrt{\lambda_{1} \lambda_{2}}, \lambda_{3}+\lambda_{4}-\left|\lambda_{5}\right|>-\sqrt{\lambda_{1} \lambda_{2}}
$$

- Perturbativity and unitarity.

We adopt a general perturbativity condition of $\left|\lambda_{i}\right| \leq 4 \pi$, and for the unitarity bound $[137-141]$ :

$$
\begin{array}{r}
\left|3\left(\lambda_{1}+\lambda_{2}\right) \pm \sqrt{9\left(\lambda_{1}-\lambda_{2}\right)^{2}+4\left(2 \lambda_{3}+\lambda_{4}\right)^{2}}\right|<16 \pi, \\
\left|\left(\lambda_{1}+\lambda_{2}\right) \pm \sqrt{\left(\lambda_{1}-\lambda_{2}\right)^{2}+4 \lambda_{4}^{2}}\right|<16 \pi, \\
\left|\left(\lambda_{1}+\lambda_{2}\right) \pm \sqrt{\left(\lambda_{1}-\lambda_{2}\right)^{2}+4 \lambda_{5}^{2}}\right|<16 \pi, \\
\left|\lambda_{3}+2 \lambda_{4} \pm 3 \lambda_{5}\right|<8 \pi, \quad\left|\lambda_{3} \pm \lambda_{4}\right|<8 \pi, \quad\left|\lambda_{3} \pm \lambda_{5}\right|<8 \pi .
\end{array}
$$

To provide some general insights into the impact of these theoretical constraints, we show in figure 1 the allowed regions in the $m_{H}-\Delta m$ (left), $m_{H}-\tan \beta$ (middle), and $\lambda v^{2}-\tan \beta$ (right) planes, for various fixed values of the other parameters. In the left panel, we take $\tan \beta=3, \cos (\beta-\alpha)=0$, fixing $m_{A}=m_{H^{ \pm}}$. Here $\sqrt{\lambda v^{2}}=0,150,300,220,230 \mathrm{GeV}$ are represented by the red, blue, green, purple, and orange lines, and the region under the lines is allowed by the theoretical constraints. Generally, a larger heavy Higgs mass $m_{H}$ corresponds to a smaller allowed mass splitting $\Delta m$ for any specific $\sqrt{\lambda v^{2}}$. The allowed $\Delta m$ also gets smaller when $\sqrt{\lambda v^{2}}$ gets larger, and here there is no region left for $\sqrt{\lambda v^{2}}>232 \mathrm{GeV}$. 
In the middle panel with $\sqrt{\lambda v^{2}}=0 \mathrm{GeV}$, we explore the effect of the parameter $\cos (\beta-\alpha)$. Here, based on the allowed $|\cos (\beta-\alpha)|$ at the current LHC Run-II [142], we take $\cos (\beta-\alpha)= \pm 0.005$ (dashed lines), and $\cos (\beta-\alpha)= \pm 0.02$ (solid lines) and show the allowed region, which is to the left of the corresponding lines. We fix the mass splitting $\Delta m=m_{A / H^{ \pm}}-m_{H}=200 \mathrm{GeV} \cdot{ }^{3}$ Under $\cos (\beta-\alpha)=0, m_{H}<820 \mathrm{GeV}$ is allowed, independently of $\tan \beta$. If $\cos (\beta-\alpha) \neq 0$, such as the 0.005 region shown by the dashed lines, the allowed regions are reduced. As discussed in [118], the allowed regions for opposite-sign $\cos (\beta-\alpha)$ are symmetric around the line $\tan \beta=1$.

In the right panel, $m_{H}$ is fixed at $700 \mathrm{GeV}$, and $\Delta m=m_{A / H^{ \pm}}-m_{H}=0,100,200$ and 230 are shown. The allowed region is inside of the boundary line. Larger $\Delta m$ leads to a smaller allowed $\lambda v^{2}$ range, and $\Delta m>230 \mathrm{GeV}$ is no longer allowed. For $\sqrt{\lambda v^{2}}=0$, there is no restriction on $\tan \beta$.

\subsection{Direct searches at LHC Run-II}

We take into account the latest heavy Higgs searches at LHC Run-II, including $A / H \rightarrow \mu \mu[143,144], A / H \rightarrow b b[145,146], A / H \rightarrow \tau \tau$ [147-149], $A / H \rightarrow \gamma \gamma$ [150-154], $A / H \rightarrow t t[155], H \rightarrow Z Z[156,157], H \rightarrow W W[158,159], A \rightarrow h Z \rightarrow b b \ell \ell[160-163]$, $A \rightarrow h Z \rightarrow \tau \tau \ell \ell[162,164,165], H \rightarrow h h$ [166-169], and $A / H \rightarrow H Z / A Z[170,171]$. To investigate the impact on the $2 \mathrm{HDM}$ parameter space of the published null results in these searches, we take the cross section times branching fraction limits, $\sigma \times \mathrm{BR}$, from the LHC studies and reinterpret them for our 2HDM model points using the SusHi package [172] to calculate the production cross-section at NNLO level, and the 2HDMC [173] code for Higgs decay branching fractions at tree level.

As a first example, taking the benchmark point $\cos (\beta-\alpha)=0, m_{12}^{2}=m_{H}^{2} \cos \beta \sin \beta$ and $m_{A}=m_{H^{+}}=m_{H}+200 \mathrm{GeV}$, we show the current collider limits in the $m_{H}-\tan \beta$ plane in figure 2, for both the Type I and Type II models. The various channels include $H / A \rightarrow b b$ (red), $H / A \rightarrow \tau \tau$ (dotted orange), $H / A \rightarrow \mu \mu$ (dot-dashed cyan), $H / A \rightarrow \gamma \gamma$ (dashed brown), $H / A \rightarrow t t$ (dot-dashed magenta) and $4 t$ production (dashed purple), as well as the exotic decay channel $A \rightarrow H Z$ (blue). Other decays such as $A \rightarrow Z h$ and $H \rightarrow h h$ will only contribute if $\cos (\beta-\alpha)$ deviates from zero at tree level [174].

For the Type-I model (left panel of figure 2), the exotic decay $A \rightarrow H Z$ channel covers $m_{H}<2 m_{t}, \tan \beta<5$ totally, and can reach to $\tan \beta=10$. Top quarks searches, $4 t+A / H \rightarrow t t$, cover $m_{H}<800 \mathrm{GeV}$ for $\tan \beta<0.3$, and $m_{H}<650 \mathrm{GeV}$ for $\tan \beta<1.1$ $A / H \rightarrow \tau \tau, \gamma \gamma$ then exclude the region $m<350 \mathrm{GeV}, \tan \beta<1$ Generally because of the $\cot \beta$-enhanced Yukawa coupling in Type-I model, only the small $\tan \beta$ region can be explored [132]. In the Type-II 2HDM (right panel), the top quark and $H / A \rightarrow \gamma \gamma$ constraints are similar to those for the Type-I model, while the fermionic decays $A / H \rightarrow b b, \tau \tau, \mu \mu$ could exclude $m_{H}$ to $800 \mathrm{GeV}$ when $\tan \beta>10$ generally. Since the $H b b$, and $H \tau \tau$ couplings are $\tan \beta$-enhanced, the $A \rightarrow H Z$ decay contributes a lot at medium and large $\tan \beta$ regions, $\tan \beta>0.5, m_{H}<2 m_{t}$ and $\tan \beta>15, m_{H}<600 \mathrm{GeV}$ Thus $m_{H}<2 m_{t}$ is totally excluded by all channels together in Type-II model, and only $1.5<\tan \beta<10$ is allowed for $m_{H}<650 \mathrm{GeV}$, which is important for our later study of the electroweak phase transition.

\footnotetext{
${ }^{3}$ In our notation, when we use $m_{A / H^{ \pm}}$, it indicates $m_{A}=m_{H^{ \pm}}$.
} 

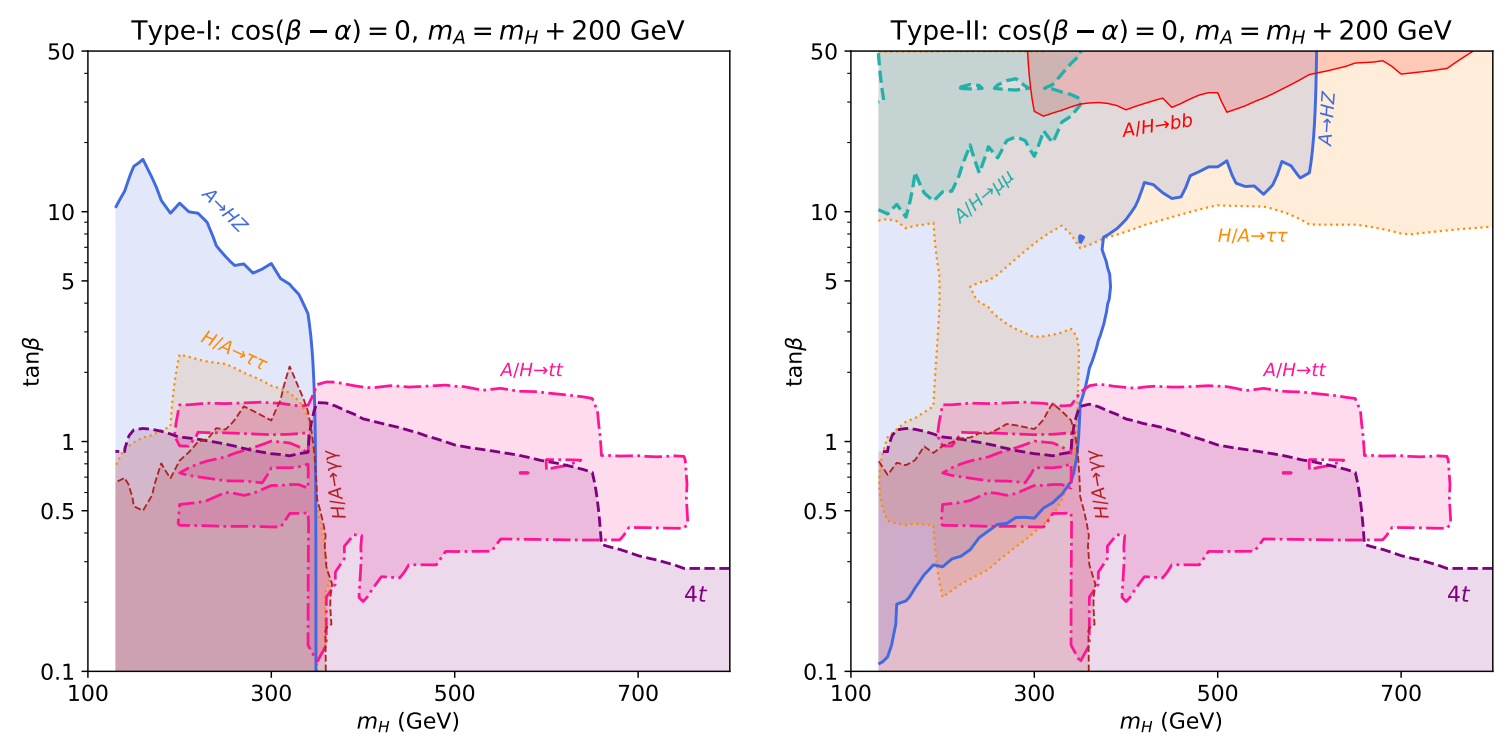

Figure 2. Direct search constraints for the heavy Higgs mass spectrum $m_{A}=m_{H^{ \pm}}=m_{H}+$ $200 \mathrm{GeV}$. We show the $95 \%$ C.L. exclusion region in the $m_{H}-\tan \beta$ plane for the Type-I 2HDM (left) and Type-II 2HDM (right) with $\cos (\beta-\alpha)=0$. The various heavy Higgs decays include i) the conventional search results on $H / A \rightarrow b b$ (red), $H / A \rightarrow \tau \tau$ (dotted orange), $H / A \rightarrow \mu \mu$ (dot-dashed cyan), $H / A \rightarrow \gamma \gamma$ (dashed brown), $H / A \rightarrow t t$ (dot-dashed magenta) and $4 t$ production (dashed purple), and ii) exotic decay channels $A \rightarrow H Z$ (blue). Other considered decays such as $A \rightarrow Z h, H \rightarrow h h$ are not relevant since their couplings are proportional to $\cos (\beta-\alpha)$.

\subsection{Higgs and $Z$ pole precision measurements}

The SM has been tested with high precision via observables measured at the $Z$-pole from LEP-I [175] and the LHC [176]. Future lepton colliders will further improve the precision of measurements in the Higgs sector, and we therefore include hypothetical future lepton collider results in our study. In ref. [177], it was shown that the precision reached by several future $e^{+} e^{-}$machines, including the CEPC program with an integrated luminosity of 5.6 $\mathrm{ab}^{-1}[108,109]$, the FCC-ee program with $5 \mathrm{ab}^{-1}$ of integrated luminosity $[115,116]$, and the ILC with various center-of-mass energies [114], is similar. Thus, following the approach adopted in refs. $[177,178]$, we will explore the CEPC proposals in detail.

In our analyses, we take the $S, T, U$ data at $95 \%$ Confidence Level (C.L.) from table 2 of ref. [177], and the Higgs precision measurements from table 3 in the same reference. We use a $\chi^{2}$ profile-likelihood fit,

$$
\chi^{2}=\sum_{i j}^{Z}\left(X_{i}-\hat{X}_{i}\right)\left(\sigma^{2}\right)_{i j}^{-1}\left(X_{j}-\hat{X}_{j}\right)+\sum_{i}^{H} \frac{\left(\mu_{i}^{2 \mathrm{HDM}}-\mu_{i}^{\mathrm{obs}}\right)^{2}}{\sigma_{\mu_{i}}^{2}},
$$

with $X_{i}=(\Delta S, \Delta T, \Delta U)_{2 \mathrm{HDM}}$ being the $2 \mathrm{HDM}$ predicted values, and $\hat{X}_{i}=(\Delta S, \Delta T, \Delta U)$ being the current best-fit central value for current measurements, and 0 for future measurements at the first term for $\mathrm{Z}$ sector. The $\sigma_{i j}$ are the error matrix, $\sigma_{i j}^{2} \equiv \sigma_{i} \rho_{i j} \sigma_{j}$ with $\sigma_{i}$ and correlation matrix $\rho_{i j}$ given in [177]. For the second term about Higgs sector, Higgs precision measurements are used to perform global fit with $\mu_{i}^{2 \mathrm{HDM}}=\left(\sigma_{i} \times \mathrm{Br}_{i}\right)^{2 \mathrm{HDM}} /\left(\sigma_{i} \times \mathrm{Br}_{i}\right)^{\mathrm{SM}}$ 
is the signal strength for various Higgs search channels, $\sigma_{\mu_{i}}$ is the estimated error for each process. The studies $[177,178]$ show that one-loop level electroweak corrections to SM Higgs couplings have probe ability to heavy Higgs with Higgs precision measurements, and thus our study of

For future colliders, the various $\mu_{i}^{\text {obs }}$ are set to be unity in the current analyses, assuming no deviations from the SM observables.

In the following analyses, the overall $\chi^{2}$ is calculated, and use to determine the allowed parameter region at the $95 \%$ C.L. For the one-, and two-parameter fits, the corresponding $\Delta \chi^{2}=\chi^{2}-\chi_{\min }^{2}$ values at the $95 \%$ C.L. are 3.84 , and 5.99 respectively.

\subsection{Flavour constraints}

The charged Higgs $H^{ \pm}$boson couples to up and down type fermions, and thus observations from flavor physics put strong bounds on its mass and couplings [179]. Among various flavor observations, measurements related to $B$ physics provide the most stringent limits on $\tan \beta$ and $m_{H^{ \pm}}$. For example, $m_{H^{ \pm}}<580 \mathrm{GeV}$ in the Type-II $2 \mathrm{HDM}$ has been excluded by the measurement of $B R\left(B \rightarrow X_{s} \gamma\right)$ [180]. $\Delta M_{B_{s}}$ and $B R\left(B_{s} \rightarrow \mu^{+} \mu^{-}\right)$further exclude $m_{H^{ \pm}}<1 \mathrm{TeV}$ in the Type-II $2 \mathrm{HDM}$ when $\tan \beta<0.7$. The region with $\tan \beta<1$ and $m_{H^{ \pm}}<1 \mathrm{TeV}$ in the Type-I $2 \mathrm{HDM}$ has been excluded by $B \rightarrow X_{s} \gamma$ [180]. In our study, we take these constraints into account.

\section{Study results}

Based on the diverse constraints above, in this section we will discuss their effects on the SFOEWPT in Type-I and Type-II 2HDMs.

\subsection{The phase transition of $2 \mathrm{HDM}$}

To get a better understanding of the electroweak phase transition in 2HDMs, we will first discuss it in the context of some approximate or limiting cases, focusing on the relationship between the phase transition and the Higgs vacuum uplifting. Then we will consider several benchmark cases, varying one or two parameters to dig into the effects of constraints as well as features of the Higgs potential. Our general results will follow these specific cases.

\subsubsection{High temperature expansion}

Due to the complicated form of the thermal effective potential eq. (2.13) and its intricate thermal evolution history, it is difficult to tell whether a specific point can successfully trigger a SFOEWPT in the early universe through a simple formula or argument. To simplify the analysis of the phase transition, people generally use a high temperature expansion, limited to the leading terms of the thermal correction functions $J_{B}$ and $J_{F}$. Then the thermal effective potential can be simplified to a polynomial function of the Higgs field value:

$$
V\left(\phi_{h}, T\right) \approx\left(D T^{2}-\mu^{2}\right) \phi_{h}^{2}-E T \phi_{h}^{3}+\frac{\tilde{\lambda}}{4} \phi_{h}^{4}
$$


Here $\phi_{h} \equiv \cos \beta \phi_{1}+\sin \beta \phi_{2}$ is the scalar field that breaks the $\mathrm{SU}(2) \times \mathrm{U}(1)$ symmetry at zero temperature. Due to the simple form of eq. (4.1), we can use the minimization condition and $V\left(0, T_{c}\right)=V\left(v_{c}, T_{c}\right)$ to directly calculate the wash-out parameter:

$$
\xi_{c} \equiv \frac{v_{c}}{T_{c}} \approx \frac{2 E}{\tilde{\lambda}}
$$

At tree-level, the coefficients $\mu^{2}$ and $\tilde{\lambda}$ in eq. (4.1) are:

$$
\mu^{2}=\frac{1}{4} m_{h}^{2}, \tilde{\lambda}=\frac{m_{h}^{2}}{2 v^{2}}
$$

The coefficients $D$ and $E$ are induced from the leading thermal corrections:

$$
\begin{aligned}
\frac{T^{4}}{2 \pi^{2}} J_{B}\left(\frac{m^{2}\left(\phi_{h}\right)}{T^{2}}\right) & \approx-\frac{\pi^{2} T^{4}}{90}+\frac{1}{24} T^{2} m^{2}\left(\phi_{h}\right)-\frac{1}{12 \pi} T\left(m^{2}\left(\phi_{h}\right)\right)^{3 / 2}+\cdots \\
\frac{T^{4}}{2 \pi^{2}} J_{F}\left(\frac{m^{2}\left(\phi_{h}\right)}{T^{2}}\right) & \approx+\frac{7}{8} \frac{\pi^{2} T^{4}}{90}-\frac{1}{48} T^{2} m^{2}\left(\phi_{h}\right)+\cdots
\end{aligned}
$$

Here $m^{2}\left(\phi_{h}\right)$ is the mass square of a massive particle with $v^{2}$ in it being replaced by $\phi_{h}^{2}$ (For example, $\left.m_{W}^{2}\left(\phi_{h}\right)=\frac{m_{W}^{2}}{v^{2}} \phi_{h}^{2}\right)$. Considering the most massive particles in the 2HDM, $D$ and $E$ can be expressed as:

$$
\begin{aligned}
& D=\frac{1}{24}\left[6 \frac{m_{W}^{2}}{v^{2}}+3 \frac{m_{Z}^{2}}{v^{2}}+\frac{m_{h}^{2}}{v^{2}}+6 \frac{m_{t}^{2}}{v^{2}}+\frac{m_{H}^{2}-M^{2}}{v^{2}}+\frac{m_{A}^{2}-M^{2}}{v^{2}}+2 \frac{m_{H^{ \pm}}^{2}-M^{2}}{v^{2}}\right] \\
& E=\frac{1}{12 \pi}\left[6 \frac{m_{W}^{3}}{v^{3}}+3 \frac{m_{Z}^{3}}{v^{3}}+\frac{m_{h}^{3}}{v^{3}}\right]+E_{\left(H / A / H^{ \pm}\right)}
\end{aligned}
$$

In the expression for $E$, the term $E_{\left(H / A / H^{ \pm}\right)}$denotes the contributions from the non-SM Higgs bosons. We cannot explicitly write out the expression for $E_{\left(H / A / H^{ \pm}\right)}$because, as we said in section 2.1, the mass of the $H / A / H^{ \pm}$bosons come from two sources. Schematically, the $\phi_{h}$-dependent non-SM Higgs squared masses can be expressed as:

$$
m_{\alpha}^{2}\left(\phi_{h}\right)=M^{2}+\lambda_{\alpha} \phi_{h}^{2}
$$

Here $M^{2}=\frac{m_{12}^{2}}{\sin \beta \cos \beta}$ is the scale at which the $\mathbb{Z}_{2}$ symmetry is broken. $\alpha$ can be $A, H$, or $H^{ \pm}$, and $\lambda_{\alpha}$ is a linear combination of the $\lambda_{i}(i=1,2,3,4,5)$ parameters. In the alignment limit $\cos (\beta-\alpha)=0$, the expressions for $\lambda_{\alpha}$ are:

$$
\begin{aligned}
\lambda_{H} & =\left(\lambda_{1}+\lambda_{2}-2 \lambda_{345}\right)\left(\sin ^{2} \beta \cos ^{2} \beta\right), \\
\lambda_{A} & =-\lambda_{5} \\
\lambda_{H^{ \pm}} & =-\frac{1}{2}\left(\lambda_{4}+\lambda_{5}\right)
\end{aligned}
$$

So the non-SM Higgs bosons provide a term in $V\left(\phi_{h}, T\right)$ which is not exactly proportional to $\phi_{h}^{3}$ :

$$
-\frac{1}{12 \pi} T\left(m_{\alpha}^{2}\left(\phi_{h}\right)\right)^{3 / 2}=-\frac{1}{12 \pi} T\left(M^{2}+\lambda_{\alpha} \phi_{h}^{2}\right)^{3 / 2}
$$


We can simplify the above expression in two limiting cases:

$$
-\frac{1}{12 \pi} T\left(M^{2}+\lambda_{\alpha} \phi_{h}^{2}\right)^{3 / 2} \approx \begin{cases}-\frac{T}{12 \pi} \lambda_{\alpha}^{3 / 2} \phi_{h}^{3}, & M^{2} \ll \lambda_{\alpha} \phi_{h}^{2} \\ -\frac{T}{12 \pi} M^{3}\left(1+\frac{3}{2} \frac{\lambda_{\alpha} \phi_{h}^{2}}{M^{2}}\right), & M^{2} \gg \lambda_{\alpha} \phi_{h}^{2}\end{cases}
$$

And so in these two limiting cases:

$$
E_{(\alpha)} \approx \begin{cases}\frac{1}{12 \pi} \lambda_{\alpha}^{3 / 2}=\frac{1}{12 \pi} \frac{m_{\alpha}^{3}}{v^{3}}, & M^{2} \ll \lambda_{\alpha} \phi_{h}^{2} \\ 0, & M^{2} \gg \lambda_{\alpha} \phi_{h}^{2}\end{cases}
$$

The above expression needs to be multiplied by 2 if $\alpha$ is $H^{ \pm}$.

Although expression (4.13) is obtained in a limiting case, it helps us to understand which of the input parameters are particularly relevant for a SPOEWPT. When the nonSM Higgs masses are dominated by $M^{2}$, the spectrum tends to be degenerate, and the phase transition strength tends to be reduced as the non-SM Higgs boson masses increase. When the non-SM Higgs masses are dominated by $\lambda_{\alpha} v^{2}$, the spectrum tends to be split, and the phase transition strength tends to be increased as the non-SM Higgs boson masses increase.

\subsubsection{Higgs vacuum uplifting}

Another method that can help us to understand which parameters are important for SFOEWPT, is to calculate the depth of the zero temperature Higgs potential [55]. For a shallow Higgs potential, it is easier to develop an energy barrier between the symmetric phase and the broken phase than for a deep Higgs potential, when the temperature is high. Thus generally speaking, there is an inverse relation between the phase transition strength and the depth of the vacuum energy. We follow the notation at ref. [55] and define the SM vacuum energy density as $\mathcal{F}_{0}^{\mathrm{SM}}$. The value of $\mathcal{F}_{0}^{\mathrm{SM}}$ is about $-1.25 \times 10^{8} \mathrm{GeV}^{4}$. The vacuum energy density of the $2 \mathrm{HDM}$ is denoted by $\mathcal{F}_{0}$. We can further define a dimensionless parameter:

$$
\Delta \mathcal{F}_{0} /\left|\mathcal{F}_{0}^{\mathrm{SM}}\right| \equiv \frac{\mathcal{F}_{0}-\mathcal{F}_{0}^{\mathrm{SM}}}{\left|\mathcal{F}_{0}^{\mathrm{SM}}\right|}
$$

$\Delta \mathcal{F}_{0} /\left|\mathcal{F}_{0}^{\mathrm{SM}}\right|>0$ means that the $2 \mathrm{HDM}$ vacuum energy is uplifted from the SM value, whilst $\Delta \mathcal{F}_{0} /\left|\mathcal{F}_{0}^{\mathrm{SM}}\right|$ cannot exceed 1 , otherwise the zero temperature vacuum will be unstable. The numerical results in [55] show a positive correlation between $\xi_{c}$ and the parameter $\Delta \mathcal{F}_{0} /\left|\mathcal{F}_{0}^{\mathrm{SM}}\right|$. However, we find that the relationship is only valid for $m_{H} \leq 500 \mathrm{GeV}$, the range ref. [55] explored, and the parameters may become negatively-correlated for large $m_{H}$. To illustrate this, here we refine their analysis by considering a benchmark case:

$$
\begin{aligned}
\tan \beta & =3.0, \\
\sqrt{\lambda v^{2}} & =0, \quad \Delta m=m_{A / H^{ \pm}}-m_{H}=200 \mathrm{GeV} .
\end{aligned}
$$

All parameters are fixed except $m_{H}$, and $\lambda v^{2}=m_{H}^{2}-\frac{m_{12}^{2}}{\sin \beta \cos \beta}=0$ (to meet the theoretical constraints, as in the right panel of figure 1). 

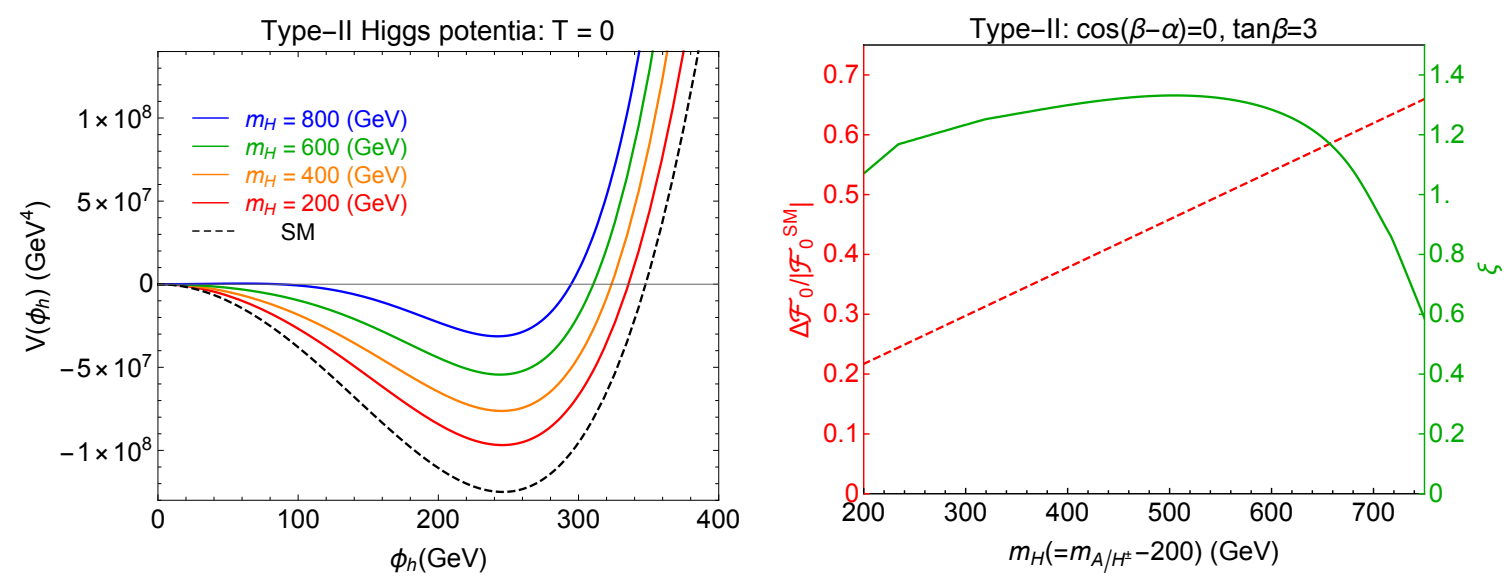

Figure 3. Zero temperature Higgs potential and its connection with the electroweak phase transition. (left): the zero temperature Higgs potential along the $\phi_{h} \equiv \cos \beta \phi_{1}+\sin \beta \phi_{2}$ direction with different $m_{H}$. (right): vacuum energy uplifting $\Delta \mathcal{F}_{0} /\left|\mathcal{F}_{0}^{\mathrm{SM}}\right|$ and wash-out parameter $\xi_{c}$ as functions of $m_{H}$.

The one-loop level Higgs vacuum uplifting in the alignment limit $\cos (\beta-\alpha)=0$ has been given in ref. [55]:

$$
\begin{aligned}
\Delta \mathcal{F}_{0}= & \frac{1}{64 \pi^{2}}\left[\left(m_{h}^{2}-2 M^{2}\right)^{2}\left(\frac{3}{2}+\frac{1}{2} \log \left[\frac{4 m_{A} m_{H} m_{H^{ \pm}}^{2}}{\left(m_{h}^{2}-2 M^{2}\right)^{2}}\right]\right)\right. \\
& \left.+\frac{1}{2}\left(m_{A}^{4}+m_{H}^{4}+2 m_{H^{ \pm}}^{4}\right)+\left(m_{h}^{2}-2 M^{2}\right)\left(m_{A}^{2}+m_{H}^{2}+2 m_{H^{ \pm}}^{2}\right)\right]
\end{aligned}
$$

To illustrate the idea underlying Higgs vacuum uplifting, here we display the whole shape of the zero temperature Higgs potential. In the left panel of figure 3, we present the zero temperature Higgs potential along the $\phi_{h} \equiv \cos \beta \phi_{1}+\sin \beta \phi_{2}$ direction, with $m_{H}=200,400,600,800 \mathrm{GeV}$ represented by red, orange, green and blue lines respectively. The SM Higgs potential is also shown with black dashed line for comparison. It is clear that as $m_{H}$ increases, the height of the minimum point of the Higgs potential continues to rise, and the shape of the Higgs potential becomes shallower. For large $m_{H}, \mathcal{F}_{0}>0$, generating an unstable vacuum. Thus for a stable vacuum $m_{H}$ cannot be too large.

To find the relationship between $\xi_{c}$ and $\Delta \mathcal{F}_{0} /\left|\mathcal{F}_{0}^{\mathrm{SM}}\right|$, in the right panel of figure 3 we present both $\Delta \mathcal{F}_{0} /\left|\mathcal{F}_{0}^{\mathrm{SM}}\right|$ and $\xi_{c}$ as functions of $m_{H}$. In the plot, the left y axis is for $\Delta \mathcal{F}_{0} /\left|\mathcal{F}_{0}^{\mathrm{SM}}\right|$ with the red dashed line representing the relationship with $m_{H}$. While $\xi_{c}$, the right y axis, is shown by the solid green line. Here $\xi_{c}$ is calculated numerically from the package BSMPT.

As the red dashed line, it is clear that there is a linear relationship between $\Delta \mathcal{F}_{0} /\left|\mathcal{F}_{0}^{\mathrm{SM}}\right|$ and $m_{H}$, similar as the left panel. But as the green line shows, $\xi_{c}$ is not monotonically dependent on $m_{H}$ and gets the maximum value around $m_{H}=500 \mathrm{GeV}$. This result can be understood by our high temperature expansion analysis. Generally as $m_{H}$, equal to $M^{2}$ in our scenario to meet theoretical constraints, becomes too large, the non-SM Higgs mass 
is dominated by $M^{2}$ and $E_{\left(H / A / H^{ \pm}\right)}$get smaller as eq. (4.13). Thus the phase transition strength becomes weaker as $m_{H}$ increases from eq. (4.6) and eq. (4.1).

Since $\Delta \mathcal{F}_{0} /\left|\mathcal{F}_{0}^{\mathrm{SM}}\right|$ always gets larger when $m_{H}$ grows, while $\xi_{c}$ gets larger at first $\left(m_{H}<500 \mathrm{GeV}\right.$ here), and then gets smaller, we can conclude $\Delta \mathcal{F}_{0} /\left|\mathcal{F}_{0}^{\mathrm{SM}}\right|$ is not monotonically correlated with $\xi_{c}$. This conclusion is different to the previous study [46].

In order to get a more robust relationship between $\Delta \mathcal{F}_{0} /\left|\mathcal{F}_{0}^{\mathrm{SM}}\right|$ and $\xi_{c}$, as well as exploring the mass splitting effects $\Delta m=m_{A / H^{ \pm}}-m_{H}$, we extend the benchmark case by including different mass splittings between the non-SM Higgs bosons:

$$
\begin{array}{rlrl}
\tan \beta & =3.0, & \cos (\beta-\alpha) & =0, \\
\sqrt{\lambda v^{2}} & =0, & m_{H} & \in(200,1000) \mathrm{GeV}, \\
\Delta m & =m_{A / H^{ \pm}}-m_{H} \in(0,300) \mathrm{GeV} .
\end{array}
$$

The reason for us to consider different mass splittings is that the mass splitting between different non-SM Higgs bosons is roughly proportional to the size of the couplings $\lambda_{i}$. Generally speaking, the greater the couplings $\lambda_{i}$, the easier it is for the non-SM Higgs bosons to change the shape of the Higgs thermal potential from eq. (4.13) and eq. (4.18). However, as can be seen from eq. (4.16), a large mass splitting tends to be more limited by vacuum stability considerations, since too large mass splitting and vacuum uplifting $\Delta \mathcal{F}_{0}$ can result to $\Delta \mathcal{F}_{0} /\left|\mathcal{F}_{0}^{\mathrm{SM}}\right|>1$. In the left panel of figure 4 , we present $\Delta \mathcal{F}_{0} /\left|\mathcal{F}_{0}^{\mathrm{SM}}\right|$ as a function of $m_{H}$ under different mass splittings $\Delta m=m_{A / H^{ \pm}}-m_{H}=50$ (red), 150 (orange), 200 (green), 250 (cyan), and 300 (blue) GeV. It is clear that the curves with the largest mass splittings quickly reach the unstable limit $\Delta \mathcal{F}_{0}=\left|\mathcal{F}_{0}^{\mathrm{SM}}\right|$ as $m_{H}$ increases. In the right panel of figure 4 , we present our scan results in the plane of $\Delta \mathcal{F}_{0} /\left|\mathcal{F}_{0}^{\mathrm{SM}}\right|-\xi_{c}$. Points with different mass splittings are tagged by different colors, with $m_{H}$ indicated by black dotted lines.

To understand our scan results, we need to invoke the analysis we performed in the last subsection. In our scenario, we have the following relationships between different parameters:

$$
\lambda_{A / H^{ \pm}} v^{2}=(\Delta m)^{2}+2 m_{H} \Delta m
$$

with $\Delta m=m_{A / H^{ \pm}}-m_{H}, m_{H}^{2}=M^{2}$. Thus, following the discussion we presented in the last subsection, if the value of $\Delta m$ is fixed and $m_{H}$ is not too large, the phase transition strength will increase as $m_{H}$ and $\Delta \mathcal{F}_{0} /\left|\mathcal{F}_{0}^{\mathrm{SM}}\right|$ increase. But if $m_{H}$ becomes too large and dominates $m_{A / H^{ \pm}}$, the phase transition strength will decrease as $m_{H}$ increases, until the vacuum becomes unstable, i.e. $\Delta \mathcal{F}_{0}=\left|\mathcal{F}_{0}^{\mathrm{SM}}\right|$. In the right panel of figure 4 , we therefore observe that $\xi_{c}$ first rises as $\Delta \mathcal{F}_{0}=\left|\mathcal{F}_{0}^{\mathrm{SM}}\right|$ increases (equivalent to $m_{H}$ increasing), and then $\xi_{c}$ decreases as $\Delta \mathcal{F}_{0}=\left|\mathcal{F}_{0}^{\mathrm{SM}}\right|$ (and $m_{H}$ ) continues to increase.

For the right panel of figure 4, depending on the mass splitting and the phase transition features, we can divide the parameter space into three regions:

1. The small mass splitting region, with mass splitting $\Delta m<160 \mathrm{GeV}$. In this case, the Higgs vacuum energy cannot be uplifted too high, which means that these points are safe from vacuum stability bounds, and $m_{H}$ can vary from $200 \mathrm{GeV}$ to $1 \mathrm{TeV}$. Due to the small value of $\Delta m$, however, $\lambda_{A / H^{ \pm}} v^{2}$ is too small to satisfy the requirement of a SFOEWPT. 

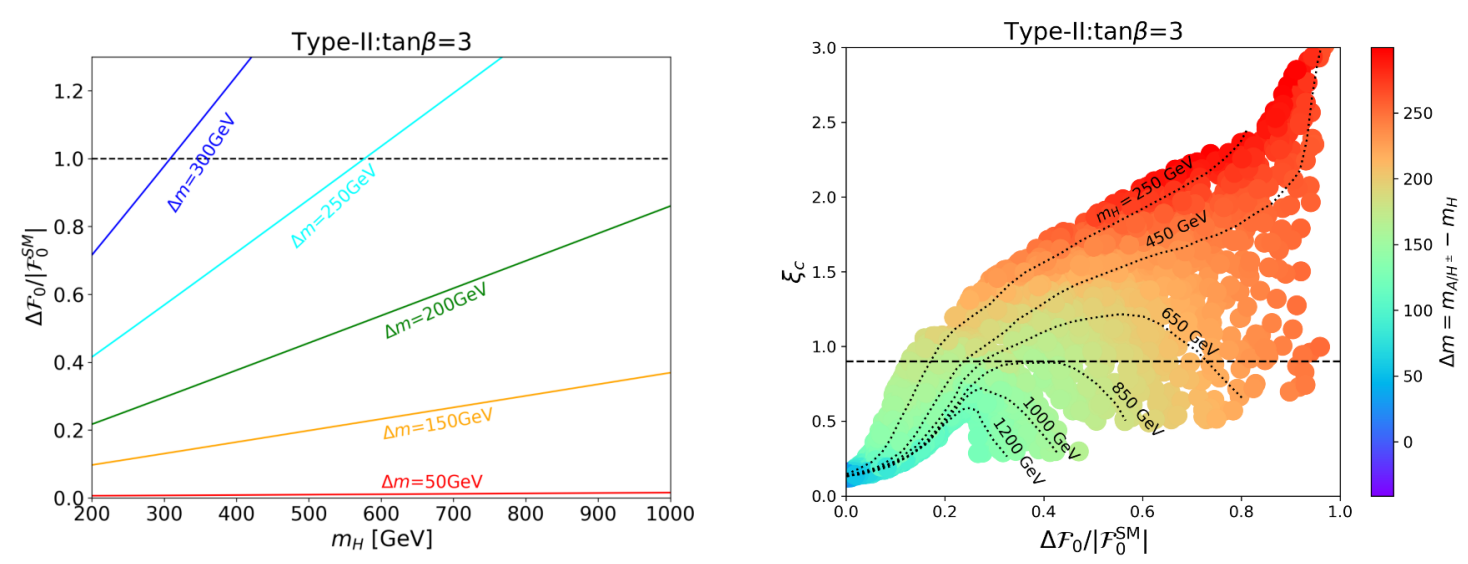

Figure 4. (left): $\Delta \mathcal{F}_{0} /\left|\mathcal{F}_{0}^{\mathrm{SM}}\right|$ as functions of $m_{H}$, with different mass splitting. (right): scan result projected in the $\Delta \mathcal{F}_{0} /\left|\mathcal{F}_{0}^{\mathrm{SM}}\right|-\xi_{c}$ plane. Points with different mass splitting are tagged by different colors, and the dotted lines mark the CP-even Higgs mass $m_{H}$.

2. The medium mass splitting region, with mass splitting $\Delta m \in[160 \mathrm{GeV}, 230 \mathrm{GeV}]$. In this case, most of the parameter space is still safe from the vacuum stability constraint. When $m_{H}$ is not too large, it helps to enhance the phase transition strength. As $m_{H}$ grows to dominate the mass expression of $m_{A / H^{ \pm}}, \xi_{c}$ rapidly decreases. We also observe that $\xi_{c}$ rises firstly and then falls as $\Delta \mathcal{F}_{0} /\left|\mathcal{F}_{0}^{\mathrm{SM}}\right|$ increases. The middle region of $\Delta \mathcal{F}_{0} /\left|\mathcal{F}_{0}^{\mathrm{SM}}\right|$ is favored by the existence of a SFOEWPT.

3. The large mass splitting region, with mass splitting $\Delta m>230 \mathrm{GeV}$. In this region $\Delta \mathcal{F}_{0} /\left|\mathcal{F}_{0}^{\mathrm{SM}}\right|$ starts from a value that is larger than 0.4 , and quickly touches the vacuum stability bound $\Delta \mathcal{F}_{0} /\left|\mathcal{F}_{0}^{\mathrm{SM}}\right|=1$ as $m_{H}$ increases. This means that, before $m_{H}$ has increased to be able to dominate $m_{A / H^{ \pm}}$, the vacuum is already unstable. We therefore observe that $\xi_{c}$ increases nearly monotonically as $\Delta \mathcal{F}_{0} /\left|\mathcal{F}_{0}^{\mathrm{SM}}\right|$ increasing.

Through the above discussion, it is clear that the upper limits on the non-SM Higgs boson masses in the 2HDM come from vacuum stability (when the mass splitting is large), or the SFOEWPT requirement (when the mass splitting is medium-large). Without the SFOEWPT requirement, the non-SM Higgs bosons can be arbitrarily heavy without violating vacuum stability, provided the mass splitting between them is small enough.

On the other hand, the black dotted lines in the right panel of figure 4 clearly show the relationship between $\xi_{c}$ and $m_{H}$. We found that $\xi_{c}$ is a monotonically increasing function of $\Delta \mathcal{F}_{0} /\left|\mathcal{F}_{0}^{\mathrm{SM}}\right|$ when $m_{H}<500 \mathrm{GeV}$, such as $m_{H}=250,450 \mathrm{GeV}$ in the right panel of figure 4. But with larger mass, the phase transition strength $\xi_{c}$ gets smaller, and when $m_{H}>850 \mathrm{GeV}, \xi_{c}$ can no longer reach 0.9 . To avoid the unstable vacuum, larger $m_{H}$ needs smaller $\Delta m$ as in figure 3. Therefore, a too large $m_{H}$ will result in a too small $\lambda_{A / H^{ \pm}} v^{2}$, which could not generate a SFOEWPT. In table 1 we present the range of $\Delta m$ and $\Delta \mathcal{F}_{0} /\left|\mathcal{F}_{0}^{\mathrm{SM}}\right|$ for different values of $m_{H}$. This clearly shows that the SFOEWPT-satisfied region keeps shrinking as $m_{H}$ gets larger and larger. 


\begin{tabular}{|c|c|c|c|c|c|}
\hline$m_{H}(\mathrm{GeV})$ & 250 & 450 & 650 & 700 & 850 \\
\hline$\Delta m(\mathrm{GeV})$ & $(170,280)$ & $(160,280)$ & $(150,230)$ & $(155,210)$ & $(160,165)$ \\
\hline$\Delta \mathcal{F}_{0} /\left|\mathcal{F}_{0}^{\mathrm{SM}}\right|$ & $(0.18,0.83)$ & $(0.25,0.95)$ & $(0.28,0.73)$ & $(0.3,0.7)$ & $(0.38,0.42)$ \\
\hline
\end{tabular}

Table 1. The parameter space for which a SFOEWPT is obtained, with $\tan \beta=3$, $\Delta m=m_{A / H^{ \pm}}-m_{H}$ in the Type-II $2 \mathrm{HDM}$, similar to figure 4.

\subsection{Case1: alignment limit with $m_{A / H^{ \pm}}-m_{H}=200 \mathrm{GeV}$}

Following the previous approximate analysis of the electroweak phase transition, we now investigate a series of benchmark cases, starting with,

$$
\begin{aligned}
& \tan \beta \in(0.2,50), \quad m_{H} \in(200,1000) \mathrm{GeV}, \\
& \cos (\beta-\alpha)=0, \quad \sqrt{\lambda v^{2}}=0, \quad \Delta m=m_{A / H^{ \pm}}-m_{H}=200 \mathrm{GeV} \text {. }
\end{aligned}
$$

Here we take $\sqrt{\lambda v^{2}}=0$ to allow for the largest range of $\tan \beta$ as shown in the second and third panel of figure 1. To explore the dependence on $m_{H}$, we fix the mass splitting $\Delta m=200 \mathrm{GeV}$, and assume the tree-level alignment limit $\cos (\beta-\alpha)=0$. The parameter space is the same as the right panel of figure 2, where there are important constraints from direct non-SM Higgs boson searches at LHC Run-II including $H / A \rightarrow \tau \tau$ (orange region with dotted line boundary, providing an upper boound), $A \rightarrow H Z$ (blue region, constraining the small mass region), and $A / H \rightarrow t t$ (red region with dash-dotted line boundary) and $4 t$ (purple region with dashed line boundary), which constrain the small $\tan \beta$ region. For the Type-II 2 HDM, there are important constraints on the mass of the charged Higgs boson from $B$ physics, which are represented by the hatched cyan dashed line. $B$ physics observables also give effective constraints at small $\tan \beta$. The hatched black line indicates the theoretical constraints, as discussed in figure 1 , requiring $m_{H}<835 \mathrm{GeV}$ for $\cos (\beta-\alpha)=0$.

After these theoretical and experimental constraints, the allowed parameter region is approximately located around $m_{H} \in(380,830) \mathrm{GeV}$, and $\tan \beta \in(1,10)$. The colored region $m_{H} \in(380,700) \mathrm{GeV}$ shows the parameter space which can generate a SFOEWPT, with dashed lines indicating the phase transition strength $\xi_{c}$. We can see that, generally, the strength $\xi_{c}$ gets its maximal value around $m_{H}=500 \mathrm{GeV}$, which is discussed in the right panel of figure 3 . The green dash-dotted lines show $\Delta \mathcal{F}_{0} /\left|\mathcal{F}_{0}^{\mathrm{SM}}\right|=0.42,0.53,0.63$, which grows with larger $m_{H}$ and is independent of $\tan \beta$. We can therefore again conclude that the SFOEWPT strength is not monotonically dependent on $m_{H}$ or $\Delta \mathcal{F}_{0} /\left|\mathcal{F}_{0}^{\mathrm{SM}}\right|$.

Finally there is a black band region round $m_{H}=700 \mathrm{GeV}$, which means that the phase transition strength $\xi_{c}<0.9$. Beside the black band region, there is a grey region which is allowed by various constraints, but $\xi_{c}$ in this region has no value. This is because the phase transition in this region is not first order, and thus we can not find the critical temperature and calculate $\xi_{c}$. We have also checked that Higgs and Z-pole precision measurements give no constraints in this case since $\cos (\beta-\alpha)=0$ and $\Delta m=200 \mathrm{GeV}$. 


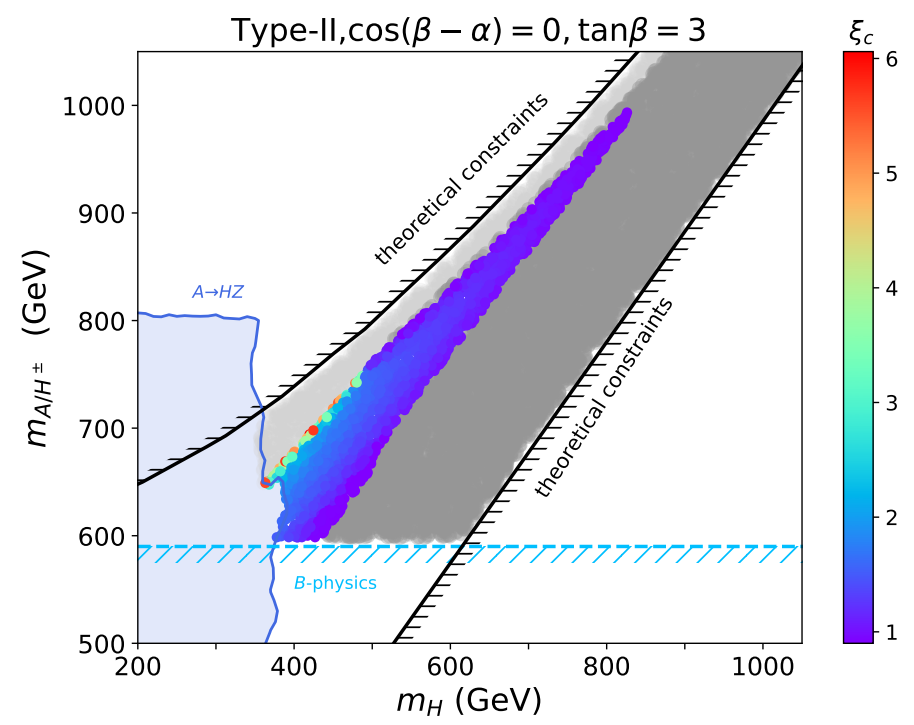

Figure 5. Electroweak phase transition and other constraints analyzed in the plane of $m_{H}-m_{A / H^{ \pm}}$, with $\cos (\beta-\alpha)=0$ and $\tan \beta=3$, for the Type-II 2HDM. Of the various heavy Higgs search channels, only $A \rightarrow H Z$ gives visible constraints, shown by the blue region. Again the hatched cyan dashed line shows the $B$-physics constraints, and the hatched black lines are for theoretical constraints. The allowed regions are divided into three parts, the colorful region with $\xi_{c}>0.9$, the light grey region (mostly above the colorful region) with $\xi_{c}<0.9$, and the dark grey region in which a SFOEWPT cannot occur.

\subsection{Case2: alignment limit with $m_{A}=m_{H^{ \pm}}$}

Based on the results in figure 4 , here we show our second benchmark case, the alignment limit with fixed $\tan \beta$,

$$
\begin{aligned}
& m_{A / H^{ \pm}} \in(500,1200) \mathrm{GeV}, \quad m_{H} \in(200,1000) \mathrm{GeV}, \\
& \cos (\beta-\alpha)=0, \quad \sqrt{\lambda v^{2}}=0, \quad \tan \beta=3 .
\end{aligned}
$$

Again here $\sqrt{\lambda v^{2}}=0$ is set to avoid the constraints on the parameter $\tan \beta$. In figure 5 , we show the constraints arising from the requirement of a SFOEWPT and other observables in the $m_{H}-m_{A / H^{ \pm}}$plane of the Type-II 2HDM. For the various heavy Higgs search channels, only $A \rightarrow H Z$ gives a visible constraint (shown by the blue region), which can exclude the region with $m_{H}<350 \mathrm{GeV}, m_{A / H^{ \pm}}<800 \mathrm{GeV}$. $B$-physics constraints, shown by the hatched cyan dashed line, exclude $m_{H^{ \pm}}<580 \mathrm{GeV}$. Since here we have $m_{A}=m_{H^{ \pm}}$ and $\cos (\beta-\alpha)=0$, the Higgs and Z-pole precision constraints are satisfied automatically. On the other hand, the theoretical constraints, indicated by hatched black lines, give a strong limit on the mass splitting range, roughly $\Delta m=m_{A / H^{ \pm}}-m_{H} \in(-50,200) \mathrm{GeV}$.

The allowed regions are divided into three parts, the colorful region with $\xi_{c}>0.9$, the light grey region which is mostly above the colorful region with $\Delta m=m_{A / H^{ \pm}}-m_{H} \approx 200 \mathrm{GeV}$.) with $\xi_{c}<0.9$, and the dark grey region in which a phase transition cannot occur. From the colored region, we find that, both a too large or 


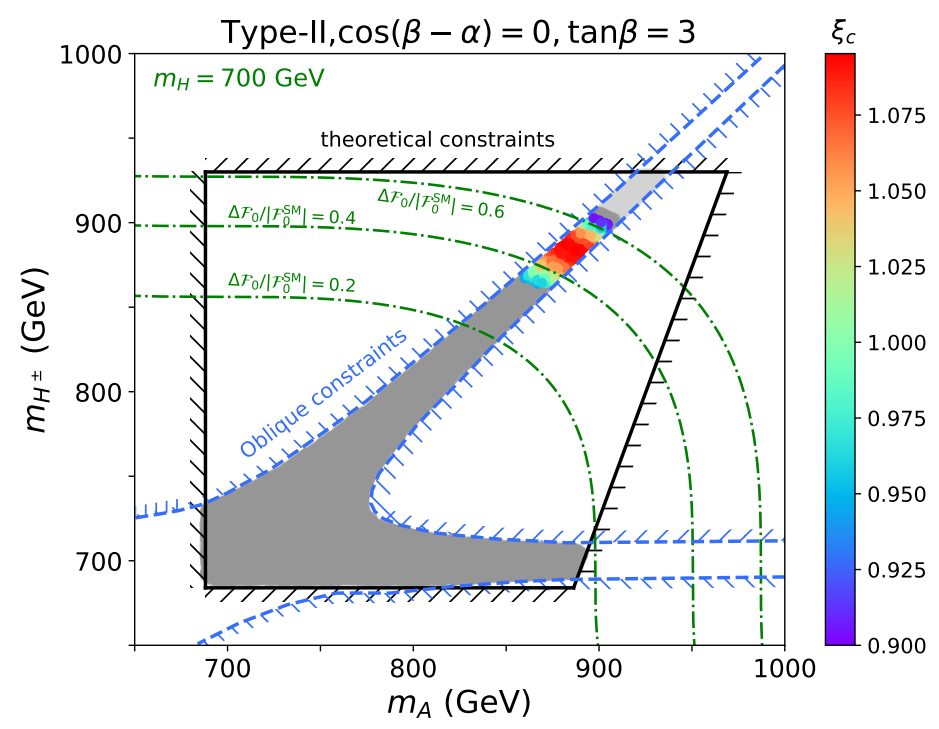

Figure 6. Electroweak phase transition and other constraints analyzed in the plane of $m_{A}-m_{H^{ \pm}}$, with $\cos (\beta-\alpha)=0$ and $\tan \beta=3$, in the Type-II 2HDM. For the various new physics search channels, only the oblique constraints make an important contribution, represented by the hatched blue dashed lines. Theoretical constraints are shown by the hatched black lines. The allowed regions are divided into three parts, the colorful region with $\xi_{c}>0.9$, the light grey region (mostly above the colorful region) with $\xi_{c}<0.9$, and the dark grey region where a first order phase transition does not occur. We also show green dash-dotted lines for $\Delta \mathcal{F}_{0} /\left|\mathcal{F}_{0}^{\mathrm{SM}}\right|$.

too small $\Delta m$ will not allow for a SFOEWPT. As discussed in figure 4 , for a too small $\Delta m$, the Higgs vacuum energy cannot be uplifted high enough to generate a phase transition, while too large a value of $\Delta m$ will result in an unstable potential $\mathcal{F}_{0}=\left|\mathcal{F}_{0}^{\mathrm{SM}}\right|$, where the potential at second EW minimal is higher than the it at the origin. This is also responsible for the upper limit on $m_{H}$, as the analysis around eq. (4.18) shows, since too small a value of $m_{H} \Delta m$ cannot generate a proper barrier for a SFOEWPT.

\subsection{Case3: alignment limit with $m_{H}=700 \mathrm{GeV}$}

In our previous case studies, we always had the simple assumption of $m_{A}=m_{H^{ \pm}}$to satisfy the oblique constraints from Z-pole measurements, and also to simplify the parameter space. Here to study the general mass splitting region, we take another benchmark case,

$$
\begin{aligned}
m_{A} & \in(500,1200) \mathrm{GeV}, & m_{H^{ \pm}} & \in(500,1200) \mathrm{GeV}, \\
m_{H} & =700 \mathrm{GeV}, & \cos (\beta-\alpha) & =0,
\end{aligned}
$$

$\sqrt{\lambda v^{2}}=0$ is once more set to avoid the constraints on the parameter $\tan \beta$, and we take $m_{H}=700 \mathrm{GeV}$ as an example. In figure 6 , we show the electroweak phase transition and other constraints in the plane of $m_{A}-m_{H^{ \pm}}$in the Type-II 2HDM. The theoretical constraints are now particularly important, as the region with hatched black lines acts as a boundary on the allowed parameter space. The lower limits on both $m_{A}$ and $m_{H^{ \pm}}$are approximately $670 \mathrm{GeV}$, while the upper limits are 970 and $930 \mathrm{GeV}$ respectively. This is because, once there is a large mass splitting, $\lambda_{1-5}$ will be enlarged [178]. For the various 
new physics search channels, only the oblique constraints make an effect here. As the hatched blue dashed lines show, the allowed regions are around either $m_{A}=m_{H^{ \pm}}$or $m_{H^{ \pm}}=m_{H}=700 \mathrm{GeV}$.

The allowed regions are divided into three parts, the colorful region with $\xi_{c}>0.9$ allowing a SFOEWPT, the light grey region (mostly above the colorful region) with $\xi_{c}<0.9$, and the dark grey region without a first order phase transition. To understand the features here, we also have green dash-dotted lines for $\Delta \mathcal{F}_{0} /\left|\mathcal{F}_{0}^{\mathrm{SM}}\right|$, which gets large when $m_{A}, m_{H^{ \pm}}$increases. We also note that, to get a proper vacuum energy uplifting, at least one of $m_{A}$ or $m_{H^{ \pm}}$should be large. For instance, $\mathcal{F}_{0} /\left|\mathcal{F}_{0}^{\mathrm{SM}}\right|=0.4$ requires $m_{H^{ \pm}}=900 \mathrm{GeV}$ when $m_{A}=m_{H}$, or $m_{A}=950 \mathrm{GeV}$ when $m_{A}=m_{H^{ \pm}}$, or $m_{A} \approx m_{H^{ \pm}} \approx 870 \mathrm{GeV}$. The region with $\xi_{c}>0.9$ is located at $\mathcal{F}_{0} /\left|\mathcal{F}_{0}^{\mathrm{SM}}\right| \in(0.37,0.63)$. The large mass limit comes from $\mathcal{F}_{0} /\left|\mathcal{F}_{0}^{\mathrm{SM}}\right| \rightarrow 1$, where the vacuum is not stable, while the small mass limit comes from eq. (4.18), where there is only limited vacuum uplifting and a barrier to generating a SFOEWPT.

\subsection{General results}

During the last section, we presented three benchmark cases to discuss the effects of the heavy Higgs masses on the existence of a SFOEWPT in the alignment limit, as well as the influence of a variety of theoretical and current experimental constraints up to the one-loop level.

In this section, we present a more general study of Type-I and Type-II 2HDMs. At the same time, we will explore the impact of future results from Higgs factories, presented in section 3.3, taking the CEPC precision measurements as an example.

Our parameter scan regions for both Type-I and Type-II are:

$$
\begin{aligned}
|\alpha|<\frac{\pi}{2}, \quad \tan \beta \in(0.2,50), & m_{A} \in(10,1500) \mathrm{GeV}, & m_{H^{ \pm}} \in(10,1500) \mathrm{GeV}, \\
m_{12}^{2} \in\left(0,1500^{2}\right) \mathrm{GeV}^{2}, & m_{h}=125.1 \mathrm{GeV}, & m_{H} \in(130,1500) \mathrm{GeV} .
\end{aligned}
$$

We perform a random parameter scan in the above parameter region, with the total number of samples exceeding 1 billion, for both Type-I and Type-II models.

In figure 8 we show the scan results for the Type-II 2HDM. The grey scatter points are the regions allowed by $B$ physics, theoretical constraints, heavy Higgs direct searches and SM Higgs precision measurements at the current LHC Run-II, and constraints from EW oblique operators. The green points are a subset of the grey ones, which can generate a SFOEWPT, and the red points are further required to meet the constraints from future Higgs precision measurements at CEPC. Compared to Case 1 (figure 7), which assumed the alignment limit and set $m_{H^{ \pm}}=m_{A}$, here we could divide the whole allowed region into 4 classes,

- Class A: regions with $m_{H}<350 \mathrm{GeV}$. Here the region has $m_{H^{ \pm}} \approx m_{A}>m_{H}$, and the mass splitting is about $(300,500) \mathrm{GeV}$ to meet the constraint $m_{H^{ \pm}}>580 \mathrm{GeV}$. Generally $\sqrt{\lambda v^{2}} \approx 0$ to allow for such a large mass splitting and $\tan \beta$ is within the region selected by the theoretical constraints shown in figure 1 . This region can also be divided into two subgroups based on $\operatorname{sign}\left(\kappa_{\mathrm{b}}\right)$. When $\operatorname{sign}\left(\kappa_{\mathrm{b}}\right)=+$, 


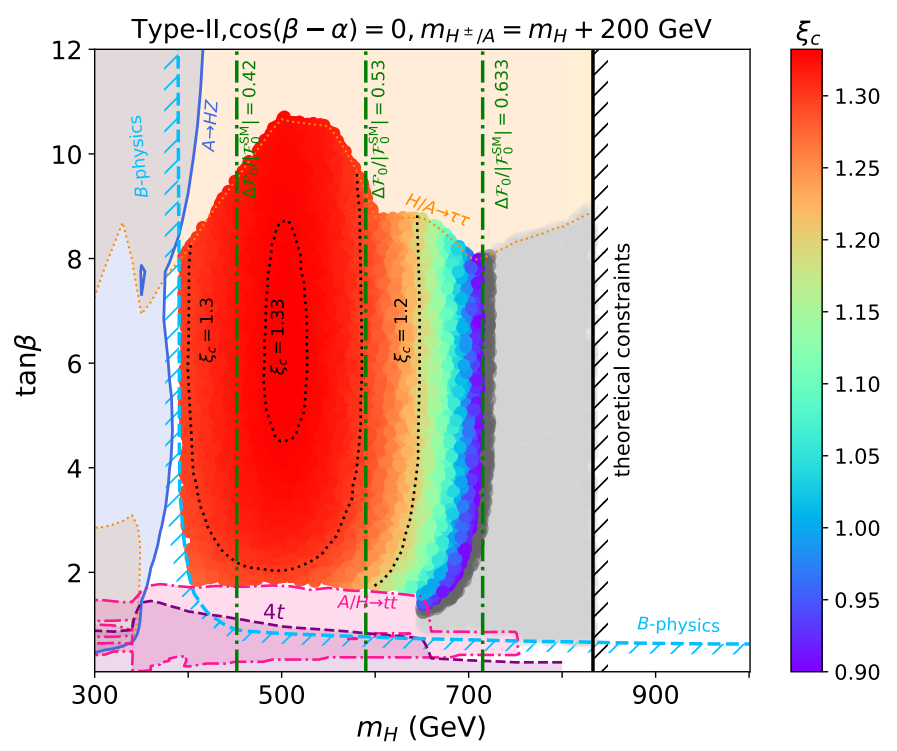

Figure 7. Electroweak phase transition and other constraints analyzed in the plane of $m_{H}-\tan \beta$ for the Type-II 2HDD. Here we fix $\Delta m=m_{A / H^{ \pm}}-m_{H}=200 \mathrm{GeV}$ and assume the tree-level alignment limit $\cos (\beta-\alpha)=0$, which is same as the right panel of figure 2 . The central colored region is allowed after all constraints. The null results of $H / A \rightarrow \tau \tau$ searches (orange region with dotted line boundary) provide an upper bound on $\tan \beta$ for a given $m_{H}$, the $A \rightarrow H Z$ results (blue region) constrain the small mass region, and the $A / H \rightarrow t t$ (red region with dash-dotted line boundary) and $4 t$ (purple region with dashed line boundary) channels constrain the small $\tan \beta$ region. The hatched cyan dashed line show the constraints from $B$ physics observables, and the hatched black line indicates the theoretical constraints. The gradient-filled regions show the parameter space that can generate a SFOEWPT, with the black dashed lines indicating the phase transition strength $\xi_{c}$. The black region means there is an electroweak phase transition with $\xi_{c}<0.9$. The grey regions are allowed by various constraints, but there is no first order phase transition. We also show $\Delta \mathcal{F}_{0} /\left|\mathcal{F}_{0}^{\mathrm{SM}}\right|$ by green dash-dotted lines.

$m_{H}<200 \mathrm{GeV}, \tan \beta \in(5,10)$ can escape the constraints from the $H \rightarrow \tau \tau$ channel as in the right panel of figure 2. At the same time, the large mass splitting $m_{A}-m_{H}>450 \mathrm{GeV}$ weakens the constraint from the $A \rightarrow H Z$ channel [132]. Another subgroup is $\operatorname{sign}\left(\kappa_{\mathrm{b}}\right)=-$, the so-called wrong-sign Yukawa coupling region with $\cos (\beta-\alpha) \approx 2 / \tan \beta$. Here $m_{H}$ can reach $350 \mathrm{GeV}, \cos (\beta-\alpha) \in(0.2,0.4)$, and LHC direct searches require $\tan \beta<10$ [142]. Because of the large mass splitting in this region, $\Delta \mathcal{F}_{0} /\left|\mathcal{F}_{0}^{\mathrm{SM}}\right|$ is too large to produce a stable vacuum.

- Class B: regions with $5<\tan \beta<12$ for $m_{H} \approx 450 \mathrm{GeV}$. This region is also a wrong-sign Yukawa coupling region with $\operatorname{sign}\left(\kappa_{\mathrm{b}}\right)=-$. Generally $\sqrt{\lambda v^{2}} \approx 0$ to meet theoretical constraints, and $m_{H^{ \pm}} \approx m_{A}=m_{H}+140 \mathrm{GeV}$ with $\tan \beta<12$ to meet constraints from the $A \rightarrow H Z$ and $A / H \rightarrow \tau \tau$ channels (see figure 2). $m_{A / H^{ \pm}}-m_{H}>140 \mathrm{GeV}$ to meet $B$ physics constraints, while a larger mass splitting is not allowed by theoretical constraints even though $\sqrt{\lambda v^{2}} \approx 0$. As the right panel of figure 4 shows, because $m_{H}=450 \mathrm{GeV}$ and $\Delta m=140 \mathrm{GeV}, \Delta \mathcal{F}_{0} /\left|\mathcal{F}_{0}^{\mathrm{SM}}\right|<0.2$ is too small, thus the vacuum uplifting is too small, and there is no SFOEWPT here. 

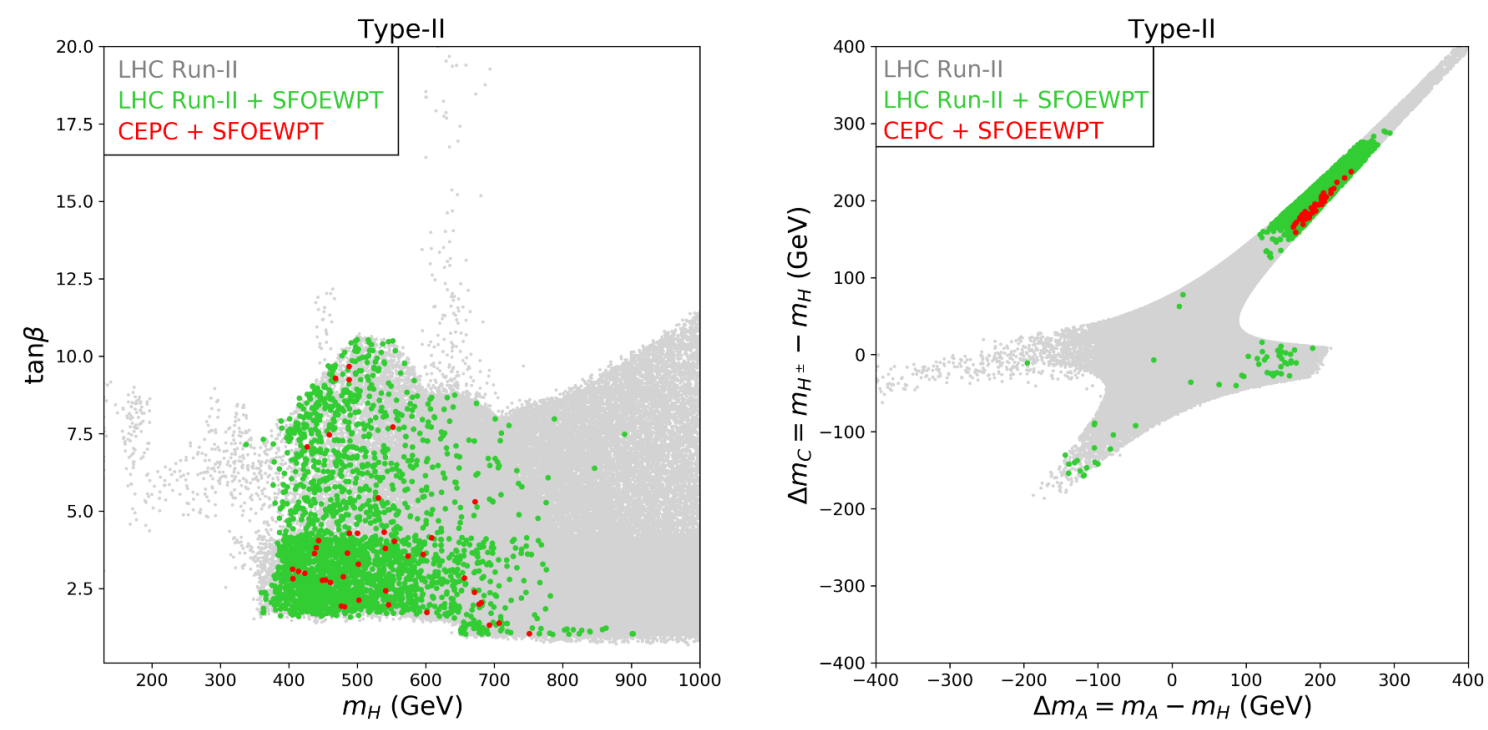

Figure 8. The allowed parameter space in the plane of $m_{H}-\tan \beta$ (left), $\Delta m_{A}-\Delta m_{C}$ (right). The grey points survive all theoretical and current experimental constraints. The green ones are able to provide a SFOEWPT, while the red ones are allowed by future precision measurements from CEPC.

- Class $C$ : regions with $5<\tan \beta<45$ for $600<m_{H}<700 \mathrm{GeV}$. Here $m_{H^{ \pm}}=m_{H}$ with $m_{A}<m_{h}=125 \mathrm{GeV}$. Again it is a wrong-sign Yukawa coupling region with $\sqrt{\lambda v^{2}} \approx 0$. The lower limit of $m_{H}$ comes from $B$ physics and EW oblique constraints, and the upper limit comes from theoretical constraints $m_{H}-m_{A}<650 \mathrm{GeV}$. In the region, $\Delta \mathcal{F}_{0} /\left|\mathcal{F}_{0}^{\mathrm{SM}}\right|<0$, thus there is no chance to generate a SFOEWPT.

- Class D: the main allowed region with $m_{H}>350 \mathrm{GeV}$. The region is similar to the white allowed region in the right panel of figure 2. Compared to Case 1 with $m_{H^{ \pm}}=m_{A}=m_{H}=200 \mathrm{GeV}$ in the alignment limit, here the allowed grey region by current LHC Run-II has no upper limit on $m_{H}$ anymore from theoretical constraints when all parameters are free. When $m_{H}<900 \mathrm{GeV}, 1<\tan \beta<10$ is required to satisfy the constraints from $H / A \rightarrow \tau \tau$, top searches and $B$ physics. When $m_{H}>900 \mathrm{GeV}, \tan \beta$ can take a larger value as the constraining power of the $H / A \rightarrow \tau \tau$ channel gets weaker. In this region, there are a number of points with $\xi_{c}>0.9$, as shown by green points. We can see the green parameter space has an upper limit of about $900 \mathrm{GeV}$. For points that also satisfy CEPC constraints as the red points, the parameter space has an upper limit of about $800 \mathrm{GeV}$.

The right panel of figure 8 shows the scan results in the plane of $\Delta m_{A}=m_{A}-m_{H}$ and $\Delta m_{C}=m_{A}-m_{H^{ \pm}}$, allowing us to analyze the Class $D$ parameter space. Here the general structure is $\Delta m_{C} \approx \Delta m_{A}$ or $\Delta m_{C}=0$ because of Z-pole oblique constraints.

For the green points from Class $D$ that satisfy LHC Run-II constraints whilst producing a SFOEWPT, there are mainly three regions. For Class D1, $\Delta m_{C} \approx \Delta m_{A} \in(100,350) \mathrm{GeV}$. The region has $m_{H} \in(350,600) \mathrm{GeV}, \sqrt{\lambda v^{2}} \approx 0$, $\tan \beta \in(1,5)$, and the features are similar to Case 3 results in section 4.4. For 


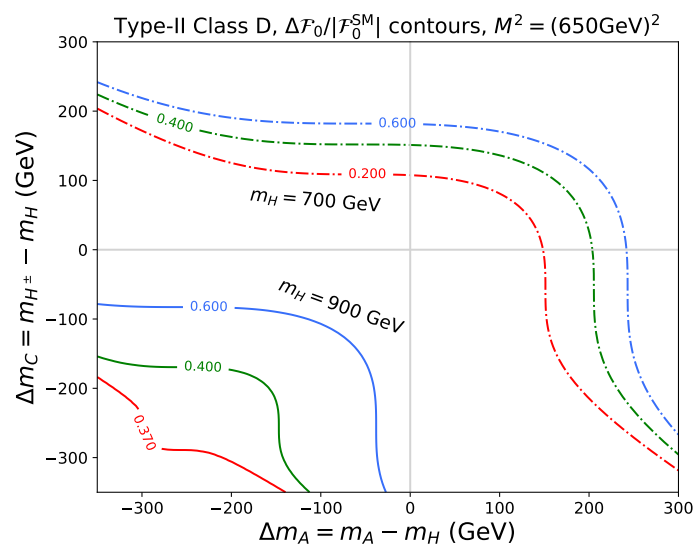

Figure 9. $\Delta \mathcal{F}_{0}\left|\mathcal{F}_{0}^{\mathrm{SM}}\right|$ contours in the plane of $\Delta m_{A}-m_{C}$ to explore Class D. Here $M^{2}=\frac{m_{12}^{2}}{\sin \beta \cos \beta}=(650 \mathrm{GeV})^{2}, m_{H}=900 \mathrm{GeV}$ (solid lines), $700 \mathrm{GeV}$ (dashed lines).

Class D2, $\Delta m_{C} \approx \Delta m_{A} \in(-200,-50) \mathrm{GeV}$. The region has $m_{H} \in(750,900) \mathrm{GeV}$, $M^{2}=\frac{m_{12}^{2}}{\sin \beta \cos \beta} \approx(650 \mathrm{GeV})^{2}, \tan \beta \approx 1$. In the left panel of figure 9 , we show the $\Delta \mathcal{F}_{0}\left|\mathcal{F}_{0}^{\mathrm{SM}}\right|$ contours in the plane of $\Delta m_{A}-m_{C}$. We can see that when $m_{H}=900 \mathrm{GeV}$ and $M=650 \mathrm{GeV}, \Delta m_{C} \approx \Delta m_{A} \in(-200,-50) \mathrm{GeV}$, which results in $\Delta \mathcal{F}_{0}\left|\mathcal{F}_{0}^{\mathrm{SM}}\right| \in$ $(0.37,0.6)$. This is one of the essential conditions for a SFOEWPT. In this region, $\sqrt{\lambda v^{2}} \in(500,600) \mathrm{GeV}$, thus theoretical constraints impose $\tan \beta \approx 1$ as shown in figure 1 . For Class D3, $\Delta m_{C} \approx 0, \Delta m_{A} \in(50,200) \mathrm{GeV}$. The region has $m_{H} \in(650,750) \mathrm{GeV}$, $M^{2}=\frac{m_{12}^{2}}{\sin \beta \cos \beta} \approx(650 \mathrm{GeV})^{2}, \tan \beta \approx 1, \sqrt{\lambda v^{2}} \in(450,550) \mathrm{GeV}$. Similarly in the left panel of figure 9 , we show the $\Delta \mathcal{F}_{0}\left|\mathcal{F}_{0}^{\mathrm{SM}}\right|$ contours for $m_{H}=700 \mathrm{GeV}$ with dash-dotted lines. Class D2 and Class D3, which are allowed by current LHC indirect Higgs precision measurements and direct heavy Higgs searches, will be excluded by Higgs precision observables at the CEPC. This is because both the Higgs precision measurements study at one-loop level and Z-pole study constrain the heavy Higgs mass splitting with sizable large $\sqrt{\lambda v^{2}}$, and the combinations give both the upper and lower limit on $\Delta m_{C}$ and $\Delta m_{A}[118,177,178]$. Here the two regions have large one-loop level corrections to the SM-like Higgs couplings, and large mass splittings around $\tan \beta=1$ are not allowed by precise measurements of the Higgs couplings.

We show our general scan results for the Type-I 2HDM in figure 10. The allowed grey, green and red points here cover a larger area than for the Type-II model, which mainly comes from heavy Higgs direct search constraints on the large $\tan \beta$ region. As the benchmark case shown in figure 2 shows, there is no constraint on $\tan \beta>2$ when $m_{H}>2 m_{t}$ in the Type-I $2 \mathrm{HDM}$ because all $H f \bar{f}$ couplings are reduced as $\tan \beta$ increases.

At the same time, there is also a larger range for $\cos (\beta-\alpha)$ at $\tan \beta>2$ compared to the Type-II 2 HDM [142]. Thus terms involving $\cos (\beta-\alpha)$ will also become important, and from ref. [55] we can get,

$$
\begin{aligned}
\left.\Delta \mathcal{F}_{0}\right|_{\text {general }}= & \left.\Delta \mathcal{F}_{0}\right|_{\cos (\beta-\alpha)=0}+\frac{1}{128 \pi^{2}} \cos (\beta-\alpha) \sin (\beta-\alpha)\left(\tan \beta-\frac{1}{\tan \beta}\right) \\
& \left(m_{H}^{2}-m_{h}^{2}\right)\left(2 m_{H^{ \pm}}^{2}+2 m_{A}^{2}+5 m_{H}^{2}-6 M^{2}\right)+\mathcal{O}\left(\cos ^{2}(\beta-\alpha)\right),
\end{aligned}
$$



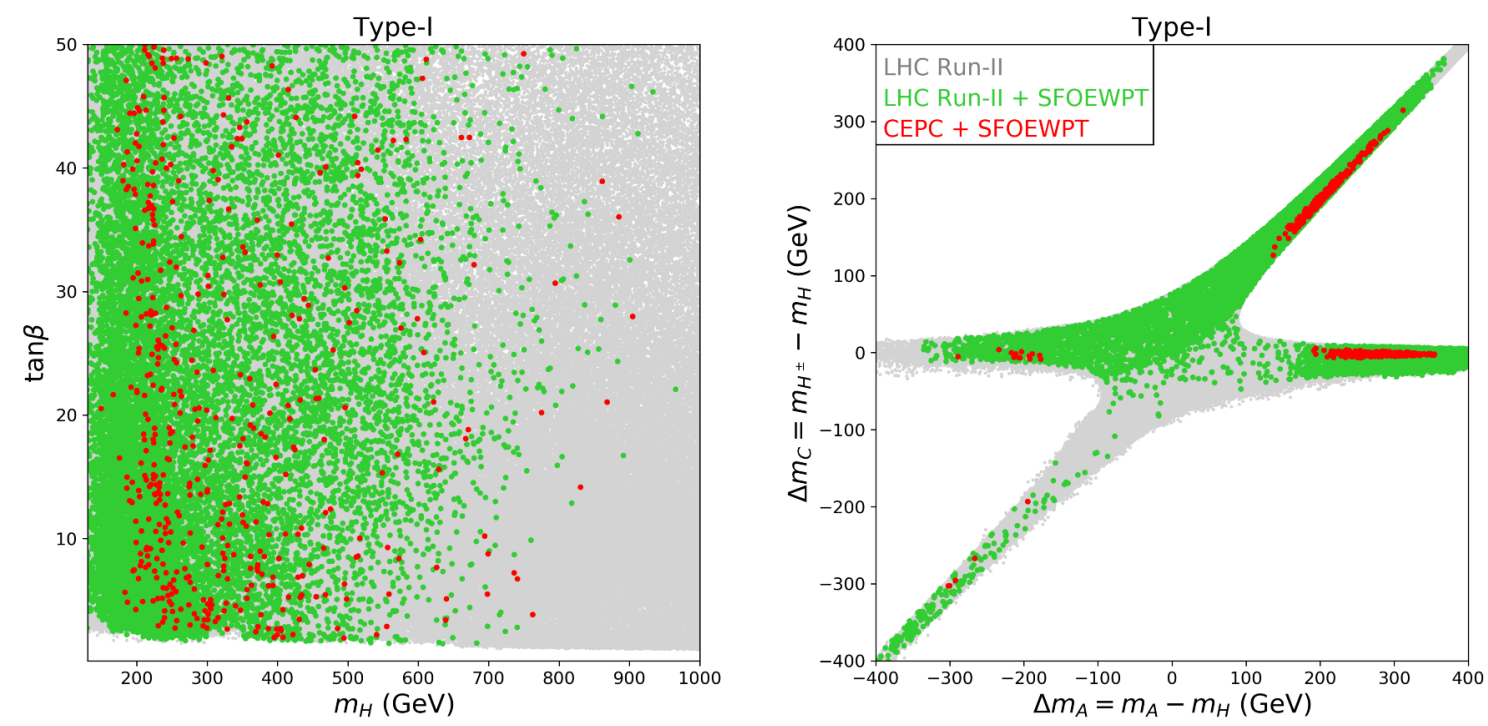

Figure 10. Allowed parameter space in the plane of $m_{H}-\tan \beta$ (left), $\Delta m_{A}-\Delta m_{C}$ (right). Same as figure 8, but for the Type-I model.

here $M^{2}=\frac{m_{12}^{2}}{\sin \beta \cos \beta}$. Because of this additional term, once there is sizable $\tan \beta, \cos (\beta-\alpha)$, the allowed $\Delta m_{A}, \Delta m_{C}$ to generate the proper $\Delta \mathcal{F}_{0} /\left|\mathcal{F}_{0}^{\mathrm{SM}}\right|$ range will be a little different to that in the Type-II case. In other words, the allowed parameter space in the Type-I model is larger than that in the Type-II model.

Generally speaking, compared to the Type-II 2HDM, the upper limit of $m_{H}$ allowed by a SFOEWPT in the Type-I model can still reach to $900 \mathrm{GeV}$. In the Type-II model, such points have $\Delta m_{A, C}<0$, and are excluded by Higgs and $Z$-pole precision measurements. But larger $\tan \beta$ values allow larger mass splittings between the heavy Higgs bosons [177, 178], and thus in the Type-I model $m_{H} \rightarrow 900 \mathrm{GeV}$ still satisfy these precision measurements. Similarly the regions with $\Delta m_{C} \approx 0, \Delta m_{A}<0$ or $\Delta m_{C} \approx 0, \Delta m_{A}>0$ which are not allowed in the Type-II model can still generate a SFOEWPT in the Type-I model.

\section{Conclusion}

In this work, we have revisited the existence of a strong first order electroweak phase transition (SFOEWPT) in the Type-I and Type-II 2HDMs Using both numerical and analytical analysis methods, we pointed out that $\Delta \mathcal{F}_{0} /\left|\mathcal{F}_{0}^{\mathrm{SM}}\right|$ is not monotonically related to $\xi_{c}$ as shown in figure 3 and figure 4 . $\xi_{c}$ grows (decreases) with larger $\Delta \mathcal{F}_{0} /\left|\mathcal{F}_{0}^{\mathrm{SM}}\right|$ for small (large) heavy mass splittings. This conclusion is different to that of a previous study [46].

We also found, SFOEWPT suggests the non-SM Higgs bosons, $H / A / H^{ \pm}$, have upper limits on their mass as our benchmark Case 1 figure 7 and general scan results figure 8 and figure 10. This limits comes from the combined requirements of vacuum stability at zero temperature and $\lambda_{H / A / H^{ \pm}} v^{2}$ corrections term at high temperature. Through Case 2 figure 5 and Case 3 figure 6, we analysed the effects of heavy Higgs mass splitting. 
After combining current bounds from LHC direct and indirect Higgs searches, current electroweak precision measurements, flavour physics, and anticipated precision measurements at the future CEPC $Z$ and Higgs factory, the requirement of SFOEWPT puts strong constraints on the mass spectrum of $H / A / H^{ \pm}$, with the allowed region:

Type-I2HDM:

$$
\begin{aligned}
& 200 \mathrm{GeV} \lesssim m_{H}, m_{A}, m_{H^{ \pm}} \lesssim 1 \mathrm{TeV}, \\
& \left|m_{A / H^{ \pm}}-m_{H}\right| \in(150,350) \mathrm{GeV} \text { or }\left|m_{A}-m_{H / H^{ \pm}}\right| \in(150,350) \mathrm{GeV}
\end{aligned}
$$

Type-II2HDM:

$$
400 \mathrm{GeV} \lesssim m_{H}, m_{A}, m_{H^{ \pm}} \lesssim 1 \mathrm{TeV}, m_{A / H^{ \pm}}-m_{H} \in(150,250) \mathrm{GeV}
$$

In Type-II 2HDM, parameter space Class D2 $\left(m_{A}=m_{H^{ \pm}}<m_{H}\right)$ and D3 $\left(m_{A}<m_{H^{ \pm}}=m_{H}\right)$ are allowed by SM Higgs precision measurements and heavy Higgs searches at LHC Run-II, but can be excluded by Higgs precision observables at the CEPC because of the one-loop level corrections to the SM-like Higgs couplings. The only allowed region Class D1 has $m_{A}=m_{H^{ \pm}}>m_{H}$ and small $\lambda v^{2}=m_{H}^{2}-\frac{m_{12}^{2}}{\sin \beta \cos \beta}$. In Type-I 2HDM, because of allowed large $\tan \beta$ region from Higgs precision measurements, Class D2 and D3 are still allowed.

Both Type-I and Type-II requires a sizable mass splitting between different heavy non-SM Higgs. And the suggested upper limits of $m_{A / H / H^{ \pm}}$is $900 \mathrm{GeV}$ at current stage, and $800 \mathrm{GeV}$ after including Higgs and Z-pole precisions at CEPC. Such a constrained spectrum points out a clear direction for direct searches at the LHC and future colliders.

\section{Acknowledgments}

We thank Martin White for useful discussion and a careful reading of the manuscript. M.Z. is supported by the National Natural Science Foundation of China (Grant No. 11947118). WS and AGW are supported by the Australian Research Council (ARC) Centre of Excellence for Dark Matter Particle Physics (CE200100008).

Open Access. This article is distributed under the terms of the Creative Commons Attribution License (CC-BY 4.0), which permits any use, distribution and reproduction in any medium, provided the original author(s) and source are credited.

\section{References}

[1] ATLAS collaboration, Observation of a new particle in the search for the Standard Model Higgs boson with the ATLAS detector at the LHC, Phys. Lett. B 716 (2012) 1 [arXiv:1207.7214] [INSPIRE].

[2] CMS collaboration, Observation of a New Boson at a Mass of $125 \mathrm{GeV}$ with the CMS Experiment at the LHC, Phys. Lett. B 716 (2012) 30 [arXiv:1207.7235] [INSPIRE].

[3] A.D. Sakharov, Violation of CP Invariance, $C$ asymmetry and baryon asymmetry of the universe, Sov. Phys. Usp. 34 (1991) 39 [INSPIRE]. 
[4] V.A. Kuzmin, V.A. Rubakov and M.E. Shaposhnikov, On the Anomalous Electroweak Baryon Number Nonconservation in the Early Universe, Phys. Lett. B 155 (1985) 36 [INSPIRE].

[5] M.E. Shaposhnikov, Possible Appearance of the Baryon Asymmetry of the Universe in an Electroweak Theory, JETP Lett. 44 (1986) 465 [INSPIRE].

[6] M.E. Shaposhnikov, Baryon Asymmetry of the Universe in Standard Electroweak Theory, Nucl. Phys. B 287 (1987) 757 [INSPIRE].

[7] N.S. Manton, Topology in the Weinberg-Salam Theory, Phys. Rev. D 28 (1983) 2019 [INSPIRE].

[8] F.R. Klinkhamer and N.S. Manton, A Saddle Point Solution in the Weinberg-Salam Theory, Phys. Rev. D 30 (1984) 2212 [InSPIRE].

[9] P. Huet and E. Sather, Electroweak baryogenesis and standard model CP-violation, Phys. Rev. D 51 (1995) 379 [hep-ph/9404302] [INSPIRE].

[10] K. Kajantie, M. Laine, K. Rummukainen and M.E. Shaposhnikov, Is there a hot electroweak phase transition at $m_{H} \geq m_{W}$ ?, Phys. Rev. Lett. 77 (1996) 2887 [hep-ph/9605288] [INSPIRE].

[11] F. Csikor, Z. Fodor and J. Heitger, Endpoint of the hot electroweak phase transition, Phys. Rev. Lett. 82 (1999) 21 [hep-ph/9809291] [INSPIRE].

[12] M. Trodden, Electroweak baryogenesis, Rev. Mod. Phys. 71 (1999) 1463 [hep-ph/9803479] [INSPIRE].

[13] T. Konstandin, Quantum Transport and Electroweak Baryogenesis, Phys. Usp. 56 (2013) 747 [arXiv: 1302.6713] [INSPIRE].

[14] M. Carena, Z. Liu and M. Riembau, Probing the electroweak phase transition via enhanced di-Higgs boson production, Phys. Rev. D 97 (2018) 095032 [arXiv: 1801.00794] [INSPIRE].

[15] J.M. Cline and K. Kainulainen, Electroweak baryogenesis and dark matter from a singlet Higgs, JCAP 01 (2013) 012 [arXiv:1210.4196] [INSPIRE].

[16] J.M. Cline, K. Kainulainen and D. Tucker-Smith, Electroweak baryogenesis from a dark sector, Phys. Rev. D 95 (2017) 115006 [arXiv:1702.08909] [INSPIRE].

[17] M. Carena, M. Quirós and Y. Zhang, Electroweak Baryogenesis from Dark-Sector CP-violation, Phys. Rev. Lett. 122 (2019) 201802 [arXiv:1811.09719] [INSPIRE].

[18] J.M. Cline, G. Laporte, H. Yamashita and S. Kraml, Electroweak Phase Transition and LHC Signatures in the Singlet Majoron Model, JHEP 07 (2009) 040 [arXiv:0905.2559] [INSPIRE].

[19] S. Profumo, M.J. Ramsey-Musolf, C.L. Wainwright and P. Winslow, Singlet-catalyzed electroweak phase transitions and precision Higgs boson studies, Phys. Rev. D 91 (2015) 035018 [arXiv: 1407.5342 ] [INSPIRE].

[20] D. Curtin, P. Meade and C.-T. Yu, Testing Electroweak Baryogenesis with Future Colliders, JHEP 11 (2014) 127 [arXiv:1409.0005] [INSPIRE].

[21] F.P. Huang and C.S. Li, Electroweak baryogenesis in the framework of the effective field theory, Phys. Rev. D 92 (2015) 075014 [arXiv: 1507.08168] [INSPIRE]. 
[22] A.V. Kotwal, M.J. Ramsey-Musolf, J.M. No and P. Winslow, Singlet-catalyzed electroweak phase transitions in the $100 \mathrm{TeV}$ frontier, Phys. Rev. D 94 (2016) 035022 [arXiv: 1605.06123] [INSPIRE].

[23] V. Vaskonen, Electroweak baryogenesis and gravitational waves from a real scalar singlet, Phys. Rev. D 95 (2017) 123515 [arXiv:1611.02073] [INSPIRE].

[24] A. Beniwal, M. Lewicki, J.D. Wells, M. White and A.G. Williams, Gravitational wave, collider and dark matter signals from a scalar singlet electroweak baryogenesis, JHEP 08 (2017) 108 [arXiv: 1702.06124] [INSPIRE].

[25] G. Kurup and M. Perelstein, Dynamics of Electroweak Phase Transition In Singlet-Scalar Extension of the Standard Model, Phys. Rev. D 96 (2017) 015036 [arXiv:1704.03381] [INSPIRE].

[26] C.-W. Chiang, M.J. Ramsey-Musolf and E. Senaha, Standard Model with a Complex Scalar Singlet: Cosmological Implications and Theoretical Considerations, Phys. Rev. D 97 (2018) 015005 [arXiv: 1707.09960] [INSPIRE].

[27] A. Alves, T. Ghosh, H.-K. Guo, K. Sinha and D. Vagie, Collider and Gravitational Wave Complementarity in Exploring the Singlet Extension of the Standard Model, JHEP 04 (2019) 052 [arXiv: 1812.09333] [InSPIRE].

[28] H.-L. Li, M. Ramsey-Musolf and S. Willocq, Probing a scalar singlet-catalyzed electroweak phase transition with resonant di-Higgs boson production in the $4 b$ channel, Phys. Rev. D 100 (2019) 075035 [arXiv: 1906. 05289] [INSPIRE].

[29] N.F. Bell, M.J. Dolan, L.S. Friedrich, M.J. Ramsey-Musolf and R.R. Volkas, Electroweak Baryogenesis with Vector-like Leptons and Scalar Singlets, JHEP 09 (2019) 012 [arXiv: 1903.11255] [INSPIRE].

[30] B. Grzadkowski and D. Huang, Spontaneous CP-Violating Electroweak Baryogenesis and Dark Matter from a Complex Singlet Scalar, JHEP 08 (2018) 135 [arXiv:1807.06987] [INSPIRE].

[31] F.P. Huang, Z. Qian and M. Zhang, Exploring dynamical CP-violation induced baryogenesis by gravitational waves and colliders, Phys. Rev. D 98 (2018) 015014 [arXiv:1804.06813] [INSPIRE].

[32] A.I. Bochkarev, S.V. Kuzmin and M.E. Shaposhnikov, Electroweak baryogenesis and the Higgs boson mass problem, Phys. Lett. B 244 (1990) 275 [INSPIRE].

[33] L.D. McLerran, M.E. Shaposhnikov, N. Turok and M.B. Voloshin, Why the baryon asymmetry of the universe is approximately $10^{-10}$, Phys. Lett. B 256 (1991) 451 [INSPIRE].

[34] A.I. Bochkarev, S.V. Kuzmin and M.E. Shaposhnikov, On the Model Dependence of the Cosmological Upper Bound on the Higgs Boson and Top Quark Masses, Phys. Rev. D 43 (1991) 369 [INSPIRE].

[35] N. Turok and J. Zadrozny, Electroweak baryogenesis in the two doublet model, Nucl. Phys. B 358 (1991) 471 [INSPIRE].

[36] A.G. Cohen, D.B. Kaplan and A.E. Nelson, Spontaneous baryogenesis at the weak phase transition, Phys. Lett. B 263 (1991) 86 [INSPIRE].

[37] N. Turok and J. Zadrozny, Phase transitions in the two doublet model, Nucl. Phys. B 369 (1992) 729 [INSPIRE]. 
[38] A.E. Nelson, D.B. Kaplan and A.G. Cohen, Why there is something rather than nothing: Matter from weak interactions, Nucl. Phys. B 373 (1992) 453 [INSPIRE].

[39] K. Funakubo, A. Kakuto and K. Takenaga, The Effective potential of electroweak theory with two massless Higgs doublets at finite temperature, Prog. Theor. Phys. 91 (1994) 341 [hep-ph/9310267] [INSPIRE].

[40] A.T. Davies, C.D. froggatt, G. Jenkins and R.G. Moorhouse, Baryogenesis constraints on two Higgs doublet models, Phys. Lett. B 336 (1994) 464 [InSPIRE].

[41] K. Funakubo, A. Kakuto, S. Otsuki, K. Takenaga and F. Toyoda, CP violating profile of the electroweak bubble wall, Prog. Theor. Phys. 94 (1995) 845 [hep-ph/9507452] [INSPIRE].

[42] K. Funakubo, A. Kakuto, S. Otsuki and F. Toyoda, Explicit CP breaking and electroweak baryogenesis, Prog. Theor. Phys. 96 (1996) 771 [hep-ph/9606282] [INSPIRE].

[43] J.M. Cline, K. Kainulainen and A.P. Vischer, Dynamics of two Higgs doublet CP-violation and baryogenesis at the electroweak phase transition, Phys. Rev. D 54 (1996) 2451 [hep-ph/9506284] [INSPIRE].

[44] K. Fuyuto and E. Senaha, Sphaleron and critical bubble in the scale invariant two Higgs doublet model, Phys. Lett. B 747 (2015) 152 [arXiv: 1504.04291] [INSPIRE].

[45] C.-W. Chiang, K. Fuyuto and E. Senaha, Electroweak Baryogenesis with Lepton Flavor Violation, Phys. Lett. B 762 (2016) 315 [arXiv:1607.07316] [INSPIRE].

[46] G.C. Dorsch, S.J. Huber and J.M. No, A strong electroweak phase transition in the 2HDM after LHC8, JHEP 10 (2013) 029 [arXiv: 1305.6610] [INSPIRE].

[47] G.C. Dorsch, S.J. Huber, K. Mimasu and J.M. No, Echoes of the Electroweak Phase Transition: Discovering a second Higgs doublet through $A_{0} \rightarrow Z H_{0}$, Phys. Rev. Lett. 113 (2014) 211802 [arXiv:1405.5537] [INSPIRE].

[48] J.M. Cline and P.-A. Lemieux, Electroweak phase transition in two Higgs doublet models, Phys. Rev. D 55 (1997) 3873 [hep-ph/9609240] [inSPIRE].

[49] L. Fromme, S.J. Huber and M. Seniuch, Baryogenesis in the two-Higgs doublet model, JHEP 11 (2006) 038 [hep-ph/0605242] [INSPIRE].

[50] J.M. Cline, K. Kainulainen and M. Trott, Electroweak Baryogenesis in Two Higgs Doublet Models and B meson anomalies, JHEP 11 (2011) 089 [arXiv:1107.3559] [INSPIRE].

[51] G.C. Dorsch, S.J. Huber, T. Konstandin and J.M. No, A Second Higgs Doublet in the Early Universe: Baryogenesis and Gravitational Waves, JCAP 05 (2017) 052 [arXiv: 1611.05874] [INSPIRE].

[52] P. Basler, M. Krause, M. Muhlleitner, J. Wittbrodt and A. Wlotzka, Strong First Order Electroweak Phase Transition in the CP-Conserving 2HDM Revisited, JHEP 02 (2017) 121 [arXiv: 1612.04086] [INSPIRE].

[53] A. Haarr, A. Kvellestad and T.C. Petersen, Disfavouring Electroweak Baryogenesis and a hidden Higgs in a CP-violating Two-Higgs-Doublet Model, arXiv:1611.05757 [INSPIRE].

[54] K. Fuyuto, W.-S. Hou and E. Senaha, Electroweak baryogenesis driven by extra top Yukawa couplings, Phys. Lett. B 776 (2018) 402 [arXiv: 1705.05034] [INSPIRE].

[55] G.C. Dorsch, S.J. Huber, K. Mimasu and J.M. No, The Higgs Vacuum Uplifted: Revisiting the Electroweak Phase Transition with a Second Higgs Doublet, JHEP 12 (2017) 086 [arXiv: 1705.09186] [INSPIRE]. 
[56] A.L. Cherchiglia and C.C. Nishi, One-loop considerations for coexisting vacua in the CP conserving 2HDM, JHEP 11 (2017) 106 [arXiv: 1707.04595] [INSPIRE].

[57] P. Basler, M. Mühlleitner and J. Wittbrodt, The CP-Violating 2HDM in Light of a Strong First Order Electroweak Phase Transition and Implications for Higgs Pair Production, JHEP 03 (2018) 061 [arXiv:1711.04097] [INSPIRE].

[58] J.O. Andersen, T. Gorda, A. Helset, L. Niemi, T.V.I. Tenkanen, A. Tranberg et al., Nonperturbative Analysis of the Electroweak Phase Transition in the Two Higgs Doublet Model, Phys. Rev. Lett. 121 (2018) 191802 [arXiv:1711.09849] [INSPIRE].

[59] J. Bernon, L. Bian and Y. Jiang, A new insight into the phase transition in the early Universe with two Higgs doublets, JHEP 05 (2018) 151 [arXiv:1712.08430] [INSPIRE].

[60] T. Gorda, A. Helset, L. Niemi, T.V.I. Tenkanen and D.J. Weir, Three-dimensional effective theories for the two Higgs doublet model at high temperature, JHEP 02 (2019) 081 [arXiv: 1802.05056] [INSPIRE].

[61] P. Basler and M. Mühlleitner, BSMPT (Beyond the Standard Model Phase Transitions): A tool for the electroweak phase transition in extended Higgs sectors, Comput. Phys. Commun. 237 (2019) 62 [arXiv:1803.02846] [INSPIRE].

[62] L. Wang, J.M. Yang, M. Zhang and Y. Zhang, Revisiting lepton-specific 2HDM in light of muon g-2 anomaly, Phys. Lett. B 788 (2019) 519 [arXiv:1809.05857] [INSPIRE].

[63] K. Kainulainen, V. Keus, L. Niemi, K. Rummukainen, T.V.I. Tenkanen and V. Vaskonen, On the validity of perturbative studies of the electroweak phase transition in the Two Higgs Doublet model, JHEP 06 (2019) 075 [arXiv: 1904.01329] [INSPIRE].

[64] X. Wang, F.P. Huang and X. Zhang, Gravitational wave and collider signals in complex two-Higgs doublet model with dynamical CP-violation at finite temperature, Phys. Rev. D 101 (2020) 015015 [arXiv: 1909.02978] [INSPIRE].

[65] D. Borah and J.M. Cline, Inert Doublet Dark Matter with Strong Electroweak Phase Transition, Phys. Rev. D 86 (2012) 055001 [arXiv:1204.4722] [INSPIRE].

[66] J.M. Cline and K. Kainulainen, Improved Electroweak Phase Transition with Subdominant Inert Doublet Dark Matter, Phys. Rev. D 87 (2013) 071701 [arXiv:1302.2614] [INSPIRE].

[67] K. Fuyuto, J. Hisano and E. Senaha, Toward verification of electroweak baryogenesis by electric dipole moments, Phys. Lett. B 755 (2016) 491 [arXiv:1510.04485] [INSPIRE].

[68] T. Modak and E. Senaha, Electroweak baryogenesis via bottom transport, Phys. Rev. D 99 (2019) 115022 [arXiv:1811.08088] [INSPIRE].

[69] W. Chao and M.J. Ramsey-Musolf, Catalysis of Electroweak Baryogenesis via Fermionic Higgs Portal Dark Matter, arXiv:1503.00028 [INSPIRE].

[70] S. Inoue, G. Ovanesyan and M.J. Ramsey-Musolf, Two-Step Electroweak Baryogenesis, Phys. Rev. D 93 (2016) 015013 [arXiv:1508.05404] [inSPIRE].

[71] L. Niemi, H.H. Patel, M.J. Ramsey-Musolf, T.V.I. Tenkanen and D.J. Weir, Electroweak phase transition in the real triplet extension of the SM: Dimensional reduction, Phys. Rev. D 100 (2019) 035002 [arXiv: 1802.10500] [INSPIRE].

[72] M. Chala, M. Ramos and M. Spannowsky, Gravitational wave and collider probes of a triplet Higgs sector with a low cutoff, Eur. Phys. J. C 79 (2019) 156 [arXiv:1812.01901] [INSPIRE]. 
[73] R. Zhou, W. Cheng, X. Deng, L. Bian and Y. Wu, Electroweak phase transition and Higgs phenomenology in the Georgi-Machacek model, JHEP 01 (2019) 216 [arXiv:1812.06217] [INSPIRE].

[74] S.A.R. Ellis, S. Ipek and G. White, Electroweak Baryogenesis from Temperature-Varying Couplings, JHEP 08 (2019) 002 [arXiv: 1905.11994] [INSPIRE].

[75] Q.-H. Cao, F.P. Huang, K.-P. Xie and X. Zhang, Testing the electroweak phase transition in scalar extension models at lepton colliders, Chin. Phys. C 42 (2018) 023103 [arXiv: 1708.04737] [INSPIRE].

[76] F.P. Huang, P.-H. Gu, P.-F. Yin, Z.-H. Yu and X. Zhang, Testing the electroweak phase transition and electroweak baryogenesis at the LHC and a circular electron-positron collider, Phys. Rev. D 93 (2016) 103515 [arXiv:1511.03969] [INSPIRE].

[77] F.P. Huang, Y. Wan, D.-G. Wang, Y.-F. Cai and X. Zhang, Hearing the echoes of electroweak baryogenesis with gravitational wave detectors, Phys. Rev. D 94 (2016) 041702 [arXiv: 1601.01640] [INSPIRE].

[78] C. Balázs, G. White and J. Yue, Effective field theory, electric dipole moments and electroweak baryogenesis, JHEP 03 (2017) 030 [arXiv:1612.01270] [INSPIRE].

[79] J. de Vries, M. Postma, J. van de Vis and G. White, Electroweak Baryogenesis and the Standard Model Effective Field Theory, JHEP 01 (2018) 089 [arXiv:1710.04061] [INSPIRE].

[80] J.M. Cline, M. Jarvinen and F. Sannino, The Electroweak Phase Transition in Nearly Conformal Technicolor, Phys. Rev. D 78 (2008) 075027 [arXiv: 0808.1512] [InSPIRE].

[81] L. Bian, Y. Wu and K.-P. Xie, Electroweak phase transition with composite Higgs models: calculability, gravitational waves and collider searches, JHEP 12 (2019) 028 [arXiv: 1909.02014] [INSPIRE].

[82] K.-P. Xie, L. Bian and Y. Wu, Electroweak baryogenesis and gravitational waves in a composite Higgs model with high dimensional fermion representations, JHEP 12 (2020) 047 [arXiv: 2005.13552] [INSPIRE].

[83] J.M. Cline, M. Joyce and K. Kainulainen, Supersymmetric electroweak baryogenesis in the WKB approximation, Phys. Lett. B 417 (1998) 79 [Erratum ibid. 448 (1999) 321] [hep-ph/9708393] [INSPIRE].

[84] A. Menon, D.E. Morrissey and C.E.M. Wagner, Electroweak baryogenesis and dark matter in the NMSSM, Phys. Rev. D 70 (2004) 035005 [hep-ph/0404184] [INSPIRE].

[85] M. Carena, N.R. Shah and C.E.M. Wagner, Light Dark Matter and the Electroweak Phase Transition in the NMSSM, Phys. Rev. D 85 (2012) 036003 [arXiv:1110.4378] [INSPIRE].

[86] X.-J. Bi, L. Bian, W. Huang, J. Shu and P.-F. Yin, Interpretation of the Galactic Center excess and electroweak phase transition in the NMSSM, Phys. Rev. D 92 (2015) 023507 [arXiv: 1503.03749] [INSPIRE].

[87] S.V. Demidov, D.S. Gorbunov and D.V. Kirpichnikov, Split NMSSM with electroweak baryogenesis, JHEP 11 (2016) 148 [Erratum ibid. 08 (2017) 080] [arXiv:1608.01985] [INSPIRE].

[88] W. Huang, Z. Kang, J. Shu, P. Wu and J.M. Yang, New insights in the electroweak phase transition in the NMSSM, Phys. Rev. D 91 (2015) 025006 [arXiv:1405.1152] [INSPIRE]. 
[89] K. Cheung, T.-J. Hou, J.S. Lee and E. Senaha, Singlino-driven Electroweak Baryogenesis in the Next-to-MSSM, Phys. Lett. B $\mathbf{7 1 0}$ (2012) 188 [arXiv:1201.3781] [InSPIRE].

[90] C. Balázs, A. Mazumdar, E. Pukartas and G. White, Baryogenesis, dark matter and inflation in the Next-to-Minimal Supersymmetric Standard Model, JHEP 01 (2014) 073 [arXiv: 1309.5091] [INSPIRE].

[91] S.J. Huber, T. Konstandin, T. Prokopec and M.G. Schmidt, Electroweak Phase Transition and Baryogenesis in the NMSSM, Nucl. Phys. B 757 (2006) 172 [hep-ph/0606298] [INSPIRE].

[92] L. Bian, H.-K. Guo and J. Shu, Gravitational Waves, baryon asymmetry of the universe and electric dipole moment in the CP-violating NMSSM, Chin. Phys. C 42 (2018) 093106 [Erratum ibid. 43 (2019) 129101] [arXiv:1704.02488] [INSPIRE].

[93] J. Kozaczuk, S. Profumo, L.S. Haskins and C.L. Wainwright, Cosmological Phase Transitions and their Properties in the NMSSM, JHEP 01 (2015) 144 [arXiv:1407.4134] [INSPIRE].

[94] A. Katz, M. Perelstein, M.J. Ramsey-Musolf and P. Winslow, Stop-Catalyzed Baryogenesis Beyond the MSSM, Phys. Rev. D 92 (2015) 095019 [arXiv: 1509.02934] [InSPIRE].

[95] S. Akula, C. Balázs, L. Dunn and G. White, Electroweak baryogenesis in the $\mathbb{Z}_{3}$-invariant NMSSM, JHEP 11 (2017) 051 [arXiv:1706.09898] [INSPIRE].

[96] C. Lee, V. Cirigliano and M.J. Ramsey-Musolf, Resonant relaxation in electroweak baryogenesis, Phys. Rev. D 71 (2005) 075010 [hep-ph/0412354] [INSPIRE].

[97] C. Balázs, M. Carena, A. Menon, D.E. Morrissey and C.E.M. Wagner, The Supersymmetric origin of matter, Phys. Rev. D 71 (2005) 075002 [hep-ph/0412264] [INSPIRE].

[98] S. Liebler, S. Profumo and T. Stefaniak, Light Stop Mass Limits from Higgs Rate Measurements in the MSSM: Is MSSM Electroweak Baryogenesis Still Alive After All?, JHEP 04 (2016) 143 [arXiv: 1512.09172] [INSPIRE].

[99] A. Kobakhidze, L. Wu and J. Yue, Electroweak Baryogenesis with Anomalous Higgs Couplings, JHEP 04 (2016) 011 [arXiv: 1512.08922] [INSPIRE].

[100] M.J. Ramsey-Musolf, P. Winslow and G. White, Color Breaking Baryogenesis, Phys. Rev. D 97 (2018) 123509 [arXiv: 1708.07511] [InSPIRE].

[101] S. Yaser Ayazi and A. Mohamadnejad, Conformal vector dark matter and strongly first-order electroweak phase transition, JHEP 03 (2019) 181 [arXiv:1901.04168] [INSPIRE].

[102] A. Mohamadnejad, Gravitational waves from scale-invariant vector dark matter model: Probing below the neutrino-floor, Eur. Phys. J. C 80 (2020) 197 [arXiv:1907.08899] [INSPIRE].

[103] T.D. Lee, A Theory of Spontaneous T Violation, Phys. Rev. D 8 (1973) 1226 [InSPIRE].

[104] G.C. Branco, P.M. Ferreira, L. Lavoura, M.N. Rebelo, M. Sher and J.P. Silva, Theory and phenomenology of two-Higgs-doublet models, Phys. Rept. 516 (2012) 1 [arXiv:1106.0034] [INSPIRE].

[105] M. Fukugita and T. Yanagida, Baryogenesis Without Grand Unification, Phys. Lett. B 174 (1986) 45 [INSPIRE]. 
[106] I. Affleck and M. Dine, A New Mechanism for Baryogenesis, Nucl. Phys. B 249 (1985) 361 [INSPIRE].

[107] FCC collaboration, HE-LHC: The High-Energy Large Hadron Collider: Future Circular Collider Conceptual Design Report Volume 4, Eur. Phys. J. ST 228 (2019) 1109 [InSPIRE].

[108] CEPC Study Group collaboration, CEPC Conceptual Design Report: Volume 2 Physics 8 S Detector, arXiv:1811.10545 [INSPIRE].

[109] CEPC Physics-Detector Study Group collaboration, The CEPC input for the European Strategy for Particle Physics - Physics and Detector, arXiv:1901.03170 [INSPIRE].

[110] S. Li, H. Song and S. Su, Probing Exotic Charged Higgs Decays in the Type-II 2HDM through Top Rich Signal at a Future 100 TeV pp Collider, JHEP 11 (2020) 105 [arXiv: 2005. 00576] [INSPIRE].

[111] F. Kling, H. Li, A. Pyarelal, H. Song and S. Su, Exotic Higgs Decays in Type-II 2HDMs at the LHC and Future 100 TeV Hadron Colliders, JHEP 06 (2019) 031 [arXiv:1812.01633] [INSPIRE].

[112] C.-R. Chen, J. Hajer, T. Liu, I. Low and H. Zhang, Testing naturalness at 100 TeV, JHEP 09 (2017) 129 [arXiv: 1705.07743] [InSPIRE].

[113] N. Craig, J. Hajer, Y.-Y. Li, T. Liu and H. Zhang, Heavy Higgs bosons at low $\tan \beta$ : from the LHC to $100 \mathrm{TeV}$, JHEP 01 (2017) 018 [arXiv:1605.08744] [INSPIRE].

[114] P. Bambade et al., The International Linear Collider: A Global Project, arXiv:1903.01629 [INSPIRE].

[115] FCC collaboration, FCC Physics Opportunities: Future Circular Collider Conceptual Design Report Volume 1, Eur. Phys. J. C 79 (2019) 474 [INSPIRE].

[116] FCC collaboration, FCC-ee: The Lepton Collider: Future Circular Collider Conceptual Design Report Volume 2, Eur. Phys. J. ST 228 (2019) 261 [INSPIRE].

[117] ATLAS collaboration, J. Lacey, Higgs results from the combination of ATLAS and CMS, ATL-PHYS-SLIDE-2017-740.

[118] J. Gu, H. Li, Z. Liu, S. Su and W. Su, Learning from Higgs Physics at Future Higgs Factories, JHEP 12 (2017) 153 [arXiv:1709.06103] [inSPIRE].

[119] M. Laine and A. Vuorinen, Basics of Thermal Field Theory, Springer 925 (2016) [arXiv: 1701.01554] [INSPIRE].

[120] S.R. Coleman and E.J. Weinberg, Radiative Corrections as the Origin of Spontaneous Symmetry Breaking, Phys. Rev. D 7 (1973) 1888 [INSPIRE].

[121] M. Quirós, Finite temperature field theory and phase transitions, in ICTP Summer School in High-Energy Physics and Cosmology (1999) [hep-ph/9901312] [INSPIRE].

[122] P.B. Arnold and O. Espinosa, The Effective potential and first order phase transitions: Beyond leading-order, Phys. Rev. D 47 (1993) 3546 [Erratum ibid. 50 (1994) 6662] [hep-ph/9212235] [INSPIRE].

[123] G.D. Moore, Measuring the broken phase sphaleron rate nonperturbatively, Phys. Rev. D 59 (1999) 014503 [hep-ph/9805264] [INSPIRE].

[124] N.K. Nielsen, On the Gauge Dependence of Spontaneous Symmetry Breaking in Gauge Theories, Nucl. Phys. B 101 (1975) 173 [InSPIRE]. 
[125] L. Di Luzio and L. Mihaila, On the gauge dependence of the Standard Model vacuum instability scale, JHEP 06 (2014) 079 [arXiv: 1404.7450] [INSPIRE].

[126] H.H. Patel and M.J. Ramsey-Musolf, Baryon Washout, Electroweak Phase Transition and Perturbation Theory, JHEP 07 (2011) 029 [arXiv:1101.4665] [inSPIRE].

[127] M. Laine, M. Meyer and G. Nardini, Thermal phase transition with full 2-loop effective potential, Nucl. Phys. B 920 (2017) 565 [arXiv:1702.07479] [InSPIRE].

[128] M. Dine, P. Huet and R.L. Singleton Jr., Baryogenesis at the electroweak scale, Nucl. Phys. B 375 (1992) 625 [INSPIRE].

[129] C.L. Wainwright, CosmoTransitions: Computing Cosmological Phase Transition Temperatures and Bubble Profiles with Multiple Fields, Comput. Phys. Commun. 183 (2012) 2006 [arXiv: 1109.4189] [INSPIRE].

[130] P. Athron, C. Balázs, A. Fowlie and Y. Zhang, PhaseTracer: tracing cosmological phases and calculating transition properties, Eur. Phys. J. C 80 (2020) 567 [arXiv:2003.02859] [INSPIRE].

[131] T. Han, S.K. Kang and J. Sayre, Muon g- 2 in the aligned two Higgs doublet model, JHEP 02 (2016) 097 [arXiv: 1511.05162] [INSPIRE].

[132] F. Kling, S. Su and W. Su, 2HDM Neutral Scalars under the LHC, JHEP 06 (2020) 163 [arXiv: 2004.04172] [INSPIRE].

[133] N.G. Deshpande and E. Ma, Pattern of Symmetry Breaking with Two Higgs Doublets, Phys. Rev. D 18 (1978) 2574 [INSPIRE].

[134] M. Sher, Electroweak Higgs Potentials and Vacuum Stability, Phys. Rept. 179 (1989) 273 [INSPIRE].

[135] S. Nie and M. Sher, Vacuum stability bounds in the two Higgs doublet model, Phys. Lett. B 449 (1999) 89 [hep-ph/9811234] [INSPIRE].

[136] S. Kanemura, T. Kasai and Y. Okada, Mass bounds of the lightest CP even Higgs boson in the two Higgs doublet model, Phys. Lett. B 471 (1999) 182 [hep-ph/9903289] [INSPIRE].

[137] H. Huffel and G. Pocsik, Unitarity Bounds on Higgs Boson Masses in the Weinberg-Salam Model With Two Higgs Doublets, Z. Phys. C 8 (1981) 13 [InSPIRE].

[138] J. Maalampi, J. Sirkka and I. Vilja, Tree level unitarity and triviality bounds for two Higgs models, Phys. Lett. B 265 (1991) 371 [INSPIRE].

[139] S. Kanemura, T. Kubota and E. Takasugi, Lee-Quigg-Thacker bounds for Higgs boson masses in a two doublet model, Phys. Lett. B 313 (1993) 155 [hep-ph/9303263] [INSPIRE].

[140] A.G. Akeroyd, A. Arhrib and E.-M. Naimi, Note on tree level unitarity in the general two Higgs doublet model, Phys. Lett. B 490 (2000) 119 [hep-ph/0006035] [INSPIRE].

[141] I.F. Ginzburg and I.P. Ivanov, Tree-level unitarity constraints in the most general 2HDM, Phys. Rev. D 72 (2005) 115010 [hep-ph/0508020] [InSPIRE].

[142] W. Su, Probing loop effects in wrong-sign Yukawa region of 2HDM, arXiv:1910.06269 [INSPIRE].

[143] CMS collaboration, Search for MSSM Higgs bosons decaying to $\mu+\mu-$ in proton-proton collisions at $s=13 \mathrm{TeV}$, Phys. Lett. B 798 (2019) 134992 [arXiv:1907.03152] [INSPIRE]. 
[144] ATLAS collaboration, Search for scalar resonances decaying into $\mu^{+} \mu^{-}$in events with and without b-tagged jets produced in proton-proton collisions at $\sqrt{s}=13$ TeV with the ATLAS detector, JHEP 07 (2019) 117 [arXiv: 1901.08144] [INSPIRE].

[145] CMS collaboration, Search for beyond the standard model Higgs bosons decaying into a $\mathrm{b} \overline{\mathrm{b}}$ pair in pp collisions at $\sqrt{s}=13 \mathrm{TeV}$, JHEP 08 (2018) 113 [arXiv: 1805.12191] [INSPIRE].

[146] ATLAS collaboration, Search for heavy neutral Higgs bosons produced in association with $b$-quarks and decaying into b-quarks at $\sqrt{s}=13 \mathrm{TeV}$ with the ATLAS detector, Phys. Rev. D 102 (2020) 032004 [arXiv: 1907.02749] [INSPIRE].

[147] CMS collaboration, Search for additional neutral MSSM Higgs bosons in the $\tau \tau$ final state in proton-proton collisions at $\sqrt{s}=13 \mathrm{TeV}$, JHEP 09 (2018) 007 [arXiv:1803.06553] [INSPIRE].

[148] CMS collaboration, Search for a low-mass $\tau^{+} \tau^{-}$resonance in association with a bottom quark in proton-proton collisions at $\sqrt{s}=13$ TeV, JHEP 05 (2019) 210 [arXiv: 1903.10228] [INSPIRE].

[149] ATLAS collaboration, Search for heavy Higgs bosons decaying into two tau leptons with the ATLAS detector using pp collisions at $\sqrt{s}=13$ TeV, Phys. Rev. Lett. 125 (2020) 051801 [arXiv: 2002.12223] [INSPIRE].

[150] CMS collaboration, Search for a standard model-like Higgs boson in the mass range between 70 and $110 \mathrm{GeV}$ in the diphoton final state in proton-proton collisions at $\sqrt{s}=8$ and 13 TeV, Phys. Lett. B 793 (2019) 320 [arXiv:1811.08459] [INSPIRE].

[151] CMS collaboration, Search for physics beyond the standard model in high-mass diphoton events from proton-proton collisions at $\sqrt{s}=13 \mathrm{TeV}$, Phys. Rev. D 98 (2018) 092001 [arXiv: 1809.00327] [INSPIRE].

[152] ATLAS collaboration, Search for Scalar Diphoton Resonances in the Mass Range $65-600$ GeV with the ATLAS Detector in pp Collision Data at $\sqrt{s}=8 \mathrm{TeV}$, Phys. Rev. Lett. 113 (2014) 171801 [arXiv:1407.6583] [INSPIRE].

[153] ATLAS collaboration, Search for new phenomena in high-mass diphoton final states using $37 \mathrm{fb}^{-1}$ of proton-proton collisions collected at $\sqrt{s}=13 \mathrm{TeV}$ with the ATLAS detector, Phys. Lett. B 775 (2017) 105 [arXiv:1707.04147] [INSPIRE].

[154] ATLAS collaboration, T.A. collaboration, Search for resonances in the 65 to $110 \mathrm{GeV}$ diphoton invariant mass range using $80 \mathrm{fb}^{-1}$ of pp collisions collected at $\sqrt{\mathrm{s}}=13 \mathrm{TeV}$ with the ATLAS detector, ATLAS-CONF-2018-025.

[155] CMS collaboration, Search for heavy Higgs bosons decaying to a top quark pair in proton-proton collisions at $\sqrt{s}=13 \mathrm{TeV}$, JHEP 04 (2020) 171 [arXiv:1908.01115] [INSPIRE].

[156] CMS collaboration, Search for a new scalar resonance decaying to a pair of $Z$ bosons in proton-proton collisions at $\sqrt{s}=13 \mathrm{TeV}$, JHEP 06 (2018) 127 [Erratum ibid. 03 (2019) 128] [arXiv: 1804.01939] [INSPIRE].

[157] ATLAS collaboration, Search for heavy $Z Z$ resonances in the $\ell^{+} \ell^{-} \ell^{+} \ell^{-}$and $\ell^{+} \ell^{-} \nu \bar{\nu}$ final states using proton-proton collisions at $\sqrt{s}=13 \mathrm{TeV}$ with the ATLAS detector, Eur. Phys. J. $C 78$ (2018) 293 [arXiv:1712.06386] [INSPIRE]. 
[158] CMS collaboration, Search for a heavy Higgs boson decaying to a pair of $W$ bosons in proton-proton collisions at $\sqrt{s}=13$ TeV, JHEP 03 (2020) 034 [arXiv:1912.01594] [INSPIRE].

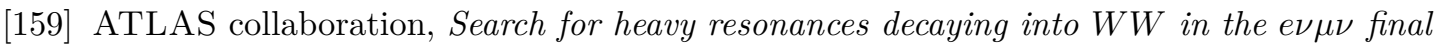
state in pp collisions at $\sqrt{s}=13$ TeV with the ATLAS detector, Eur. Phys. J. C 78 (2018) 24 [arXiv: 1710.01123] [INSPIRE].

[160] CMS collaboration, Search for a pseudoscalar boson decaying into a $Z$ boson and the 125 GeV Higgs boson in $\ell^{+} \ell^{-} b \bar{b}$ final states, Phys. Lett. B 748 (2015) 221 [arXiv:1504.04710] [INSPIRE].

[161] CMS collaboration, Search for a heavy pseudoscalar boson decaying to a $Z$ and a Higgs boson at $\sqrt{s}=13$ TeV, Eur. Phys. J. C 79 (2019) 564 [arXiv:1903.00941] [InSPIRE].

[162] ATLAS collaboration, Search for a CP-odd Higgs boson decaying to Zh in pp collisions at $\sqrt{s}=8 \mathrm{TeV}$ with the ATLAS detector, Phys. Lett. B 744 (2015) 163 [arXiv:1502.04478] [INSPIRE].

[163] ATLAS collaboration, Search for heavy resonances decaying into a $W$ or $Z$ boson and a Higgs boson in final states with leptons and b-jets in $36 \mathrm{fb}^{-1}$ of $\sqrt{\mathrm{s}}=13 \mathrm{TeV}$ pp collisions with the ATLAS detector, JHEP 03 (2018) 174 [Erratum ibid. 11 (2018) 051] [arXiv: 1712.06518] [INSPIRE].

[164] CMS collaboration, Searches for a heavy scalar boson $H$ decaying to a pair of $125 \mathrm{GeV}$ Higgs bosons hh or for a heavy pseudoscalar boson A decaying to Zh, in the final states with $h \rightarrow \tau \tau$, Phys. Lett. B 755 (2016) 217 [arXiv:1510.01181] [InSPIRE].

[165] CMS collaboration, Search for a heavy pseudoscalar Higgs boson decaying into a 125 GeV Higgs boson and a $Z$ boson in final states with two tau and two light leptons at $\sqrt{s}=13$ TeV, JHEP 03 (2020) 065 [arXiv: 1910.11634] [INSPIRE].

[166] CMS collaboration, Search for Higgs boson pair production in the bb $\tau \tau$ final state in proton-proton collisions at $\sqrt{(s)}=8 \mathrm{TeV}$, Phys. Rev. D 96 (2017) 072004 [arXiv: 1707.00350] [INSPIRE].

[167] CMS collaboration, Combination of searches for Higgs boson pair production in proton-proton collisions at $\sqrt{s}=13$ TeV, Phys. Rev. Lett. 122 (2019) 121803 [arXiv: 1811.09689] [INSPIRE].

[168] ATLAS collaboration, Searches for Higgs boson pair production in the $h h \rightarrow b b \tau \tau, \gamma \gamma W W^{*}, \gamma \gamma b b, b b b b$ channels with the ATLAS detector, Phys. Rev. D 92 (2015) 092004 [arXiv: 1509.04670] [INSPIRE].

[169] ATLAS collaboration, Combination of searches for Higgs boson pairs in pp collisions at $\sqrt{s}=13$ TeV with the ATLAS detector, Phys. Lett. B 800 (2020) 135103 [arXiv: 1906.02025] [INSPIRE].

[170] ATLAS collaboration, Search for a heavy Higgs boson decaying into a $Z$ boson and another heavy Higgs boson in the lebb final state in pp collisions at $\sqrt{s}=13$ TeV with the ATLAS detector, Phys. Lett. B 783 (2018) 392 [arXiv:1804.01126] [INSPIRE].

[171] CMS collaboration, Search for new neutral Higgs bosons through the $H \rightarrow Z A \rightarrow \ell^{+} \ell^{-} \mathrm{b} \overline{\mathrm{b}}$ process in pp collisions at $\sqrt{s}=13 \mathrm{TeV}$, JHEP 03 (2020) 055 [arXiv:1911.03781] [INSPIRE]. 
[172] S. Liebler, S. Patel and G. Weiglein, Phenomenology of on-shell Higgs production in the MSSM with complex parameters, Eur. Phys. J. C 77 (2017) 305 [arXiv:1611. 09308] [INSPIRE].

[173] D. Eriksson, J. Rathsman and O. Stal, 2HDMC: Two-Higgs-Doublet Model Calculator Physics and Manual, Comput. Phys. Commun. 181 (2010) 189 [arXiv:0902.0851] [INSPIRE].

[174] W. Su, M. White, A.G. Williams and Y. Wu, Exploring the low $\tan \beta$ region of two Higgs doublet models at the LHC, arXiv:1909.09035 [INSPIRE].

[175] ALEPH, DELPHI, L3, OPAL, SLD, LEP Electroweak Working Group, SLD Electroweak Group and SLD Heavy Flavour Group collaborations, Precision electroweak measurements on the $Z$ resonance, Phys. Rept. 427 (2006) 257 [hep-ex/0509008] [INSPIRE].

[176] J. Haller, A. Hoecker, R. Kogler, K. Mönig, T. Peiffer and J. Stelzer, Update of the global electroweak fit and constraints on two-Higgs-doublet models, Eur. Phys. J. C 78 (2018) 675 [arXiv: 1803.01853] [INSPIRE].

[177] N. Chen, T. Han, S. Li, S. Su, W. Su and Y. Wu, Type-I 2HDM under the Higgs and Electroweak Precision Measurements, JHEP 08 (2020) 131 [arXiv: 1912.01431] [INSPIRE].

[178] N. Chen, T. Han, S. Su, W. Su and Y. Wu, Type-II 2HDM under the Precision Measurements at the Z-pole and a Higgs Factory, JHEP 03 (2019) 023 [arXiv: 1808. 02037] [INSPIRE].

[179] HFLAV collaboration, Averages of b-hadron, c-hadron, and $\tau$-lepton properties as of summer 2016, Eur. Phys. J. C 77 (2017) 895 [arXiv: 1612.07233] [InSPIRE].

[180] A. Arbey, F. Mahmoudi, O. Stal and T. Stefaniak, Status of the Charged Higgs Boson in Two Higgs Doublet Models, Eur. Phys. J. C 78 (2018) 182 [arXiv:1706.07414] [InSPIRE]. 